\title{
VARIATIONAL PROBLEMS ON CONTACT RIEMANNIAN MANIFOLDS
}

\author{
SHUKICHI TANNO
}

\begin{abstract}
We define the generalized Tanaka connection for contact Riemannian manifolds generalizing one for nondegenerate, integrable CR manifolds. Then the torsion and the generalized Tanaka-Webster scalar curvature are defined properly. Furthermore, we define gauge transformations of contact Riemannian structure, and obtain an invariant under such transformations. Concerning the integral related to the invariant, we define a functional and study its first and second variational formulas. As an example, we study this functional on the unit sphere as a standard contact manifold.
\end{abstract}

\section{INTRODUCTION}

Chern-Hamilton [3] studied a kind of the Dirichlet energy concerning the Webster torsion $\tau$ of a 3-dimensional compact contact manifold, and a problem analogous to the Yamabe problem on conformal deformability to a constant scalar curvature metric. Jerison-Lee $[4,5,6]$ also obtained theorems in the setting of compact strongly pseudoconvex integrable CR manifolds, which are analogous to Aubin's theorem for the Yamabe problem. Here, the scalar curvature is the Tanaka-Webster scalar curvature ${ }^{*} S$ of a nondegenerate, integrable CR structure. Corresponding to conformal geometry in the Riemannian case, Jerison-Lee [4] defined an invariant $\lambda$ (CR invariant) for a compact strongly pseudoconvex, integrable CR manifold.

A contact manifold $(M, \eta)$ with a Riemannian metric $g$ associated with $\eta$ is called a contact Riemannian manifold. The notion of contact Riemannian structure is wider than the notion of strongly pseudo-convex, integrable $\mathrm{CR}$ structure, because the former satisfying the integrability condition $Q=0$ (cf. (2.1)) corresponds to the latter. Therefore, the research on contact Riemannian manifolds has two aspects: the first one is to study contact manifolds with the aid of associated Riemannian metrics, and the second is to give natural generalizations of results on strongly pseudoconvex, integrable CR manifolds.

Received by the editors August 7, 1987 and, in revised form, February 4, 1988.

1980 Mathematics Subject Classification (1985 Revision). Primary 53C15; Secondary 48F99.

Key words and phrases. Contact structure, Tanaka-Webster scalar curvature, gauge transformation of contact Riemannian structure.

This research was partially supported by Grant-in-Aid for Scientific Research (No. 62540021), Ministry of Education. 
The motivation of this paper is the following: Since there are so many Riemannian metrics associated with a contact form $\eta$ on a contact manifold $(M, \eta)$, we want to find some proper one among them canonically related to $\eta$. One method of finding conditions of nice Riemannian metrics is to study variational problems and their critical points. Furthermore, in the study of a contact manifold $(M, \eta)$, it is desirable to find differential geometric properties which are independent of the choice of contact forms $f \eta, f$ being positive functions on $M$. So, we study gauge transformations of contact Riemannian structure.

One basic idea is to define the canonical connection, the torsion tensor and the scalar curvature for a contact Riemannian structure, considering contact Riemannian structure as a generalization of strongly pseudoconvex, integrable CR structure. Then we can study variational problems related to the torsion or scalar curvature, and we obtain an invariant under gauge transformations of contact Riemannian structure.

$\S 1$ and $\S 2$ are devoted to preliminaries for contact Riemannian structures and CR structures. In $\S 3$ we define the generalized Tanaka connection ${ }^{*} \nabla$ for a contact Riemannian manifold generalizing the Tanaka connection for a nondegenerate, integrable CR manifold. We get the torsion tensor ${ }^{*} T$ of ${ }^{*} \nabla$. In $\S 4$, the space of all Riemannian metrics associated with a contact form on a contact manifold is explained.

In $\S 5$ we study a critical metric of the Dirichlet energy concerning the torsion tensor ${ }^{*} T$ with respect to deformations of Riemannian metrics associated with a fixed contact form. In $\S 6$ we give the relation between the torsion tensor and the generalized Webster torsion. Furthermore, as the referee kindly pointed out, the method of adapted moving frames for the complexified tangent bundle, as in Webster [18], is useful also for contact Riemannian structures. So, we give the structure equations with respect to the complex adapted frame.

In $\S 7$ we study a condition concerning the torsion tensor, because it is related to some interesting geometric properties.

In $\S 8$ we define the generalized Tanaka-Webster scalar curvature ${ }^{*} S$ for a contact Riemannian manifold, generalizing one for a nondegenerate, integrable $\mathrm{CR}$ manifold. In $\S 9$, as a generalized notion of conformal deformations in the Riemannian case, we define gauge transformations of contact Riemannian structure. In $\S 10$ we obtain the transformation law of the generalized TanakaWebster scalar curvature under gauge transformations. In $\S 11$ we obtain an invariant under gauge transformations of contact Riemannian structure. The existence of an invariant itself has an importance in the geometry of contact structures.

In $\S 12$, concerning the integral related to the invariant obtained in $\S 11$, we define a functional on the product of two spaces and study the first variation. In $\S 13$ we obtain the expression of the second variation of the functional at a critical pair. 
In $\S 14$, since the odd dimensional unit sphere is one of the standard models of contact Riemannian manifolds, we study the behavior of the functional in the neighborhood of a special critical pair, on the unit sphere.

\section{Contact Riemannian manifolds}

An $m$-dimensional manifold $M$ is a contact manifold if it admits a 1 -form $\eta^{\prime}$ such that $\eta^{\prime} \wedge\left(d \eta^{\prime}\right)^{n} \neq 0$ everywhere on $M$, where $m=2 n+1$. By a function or tensor field on $M$ we mean a smooth one. We fix a 1-form $\eta$ among $\left\{f \eta^{\prime}\right.$ : for positive functions $f$ on $\left.M\right\}$, which is called a contact form associated with the contact structure. Then we have a unique vector field $\xi$ such that

$$
\eta(\xi)=1, \quad L_{\xi} \eta=0, \quad \text { i.e., } i_{\xi} d \eta=0,
$$

where $L_{\xi}$ denotes the Lie derivation by $\xi$ and $i_{\xi}$ denotes the interior product operator by $\xi$. It is well known that there is a Riemannian metric $g$ and a $(1,1)$-tensor field $\phi$ such that

$$
g(\xi, X)=\eta(X), \quad 2 g(X, \phi Y)=d \eta(X, Y), \quad \phi \phi X=-X+\eta(X) \xi,
$$

where $X$ and $Y$ are vector fields on $M . g$ is called a Riemannian metric associated with $\eta$. The next relation follows from the above.

$$
\begin{gathered}
\phi \xi=0, \quad \eta(\phi X)=0, \\
g(X, Y)=g(\phi X, \phi X)+\eta(X) \eta(Y), \\
d \eta(X, \phi Y)=-d \eta(\phi X, Y) .
\end{gathered}
$$

Lemma 1.1. Concerning the Riemannian connection $\nabla$ with respect to $g$, the following hold:

(i) $\nabla_{\xi} \eta=0, \nabla_{\xi} \xi=0, \xi^{r} \nabla_{i} \eta_{r}=0$;

(ii) $\nabla_{r} \xi^{r}=0, \nabla_{r} \phi_{j}^{r}=-2 n \eta_{j}$

(iii) $\nabla_{r} \eta_{s} \phi_{i}^{r} \phi_{j}^{s}=-\nabla_{j} \eta_{i}$

(iii' ) $\nabla_{r} \eta_{i} \phi_{j}^{r}$ and $\nabla_{i} \eta_{r} \phi_{j}^{r}$ are symmetric in $i, j$;

(iv) $\nabla_{\xi} \phi=0$.

Lemma 1.1 is known. We give here simple proofs. (i) follows from $2 \phi_{i j}=$ $\nabla_{i} \eta_{j}-\nabla_{j} \eta_{i}, \nabla_{r}\left(\eta_{i} \xi^{i}\right)=0$ and $\xi^{i} \phi_{i j}=0$, where $\phi_{i j}=g_{i r} \phi_{j}^{r}$.

The first assertion of (ii) follows from the fact that volume element $d M$ of $(M, g)$ is equal to (a constant multiple of) $\eta \wedge(d \eta)^{n}$ and that $L_{\xi} \eta=0$ implies $L_{\xi}\left(\eta \wedge(d \eta)^{n}\right)=0$. To prove the second assertion of (ii), we first notice that $\nabla_{r} \phi_{j}^{r} \xi^{j}=-\phi^{r j} \nabla_{r} \eta_{j}=-\phi^{r j} \phi_{r j}=-2 n$ implies

$$
\left(\nabla_{r} \phi_{j}^{r}+2 n \eta_{j}\right) \xi^{j}=0 \text {. }
$$

Next we verify

$$
\left(\nabla_{r} \phi_{j}^{r}+2 n \eta_{j}\right) \phi_{k}^{j}=-\phi^{i j} \nabla_{i} \phi_{j k}=0
$$

by using the closedness of $d \eta$. Thus we get (ii). 
Since

$$
L_{\xi} \phi^{i j} \phi_{j k}=L_{\xi}\left(\phi^{i j} \phi_{j k}\right)-\phi^{i j} L_{\xi} \phi_{j k}=0 \text {, }
$$

and $L_{\xi} \phi^{i j} \eta_{j}=0$, we obtain $L_{\xi} \phi^{i j}=0$. So, $\phi^{i j}=g^{i r} g^{j s} \phi_{r s}$ implies

$$
\left(\nabla_{r} \eta_{i}+\nabla_{i} \eta_{r}\right) \phi_{j}^{r}-\left(\nabla_{r} \eta_{j}+\nabla_{j} \eta_{r}\right) \phi_{i}^{r}=0
$$

Writing down $d \eta(X, \phi Y)+d \eta(\phi X, Y)=0$ in the tensor components and adding the result to the above, we get (iii' ${ }^{\prime}$ ). (iii) follows from (iii ${ }^{\prime}$ ).

Finally (iv) follows from $L_{\xi} d \eta=0$ and (iii').

An orthonormal frame $\left\{\xi, e_{\alpha}, e_{\bar{\alpha}}\right\}$ is called an adapted frame, if $e_{\bar{\alpha}}=\phi e_{\alpha}$, where $\bar{\alpha}=\alpha+n$, and $\alpha, \beta, \ldots$ run from 1 to $n$.

For tensor fields $T=\left(T_{i j}\right)$ and $V=\left(V_{i j}\right)$ for example, we use the following notations:

$$
(T ; V)=T^{i j} V_{i j}=g^{i r} g^{j s} T_{r s} V_{i j}
$$

and $\|T\|^{2}=(T ; T)$.

For more details on basic properties of contact Riemannian manifolds, see Blair [1], Sasaki [7], Sasaki-Hatakeyama [8], Tanno [12, 13], etc.

\section{CONTACT RIEMANNIAN STRUCTURE AND CR STRUCTURE}

Let $T M$ be the tangent bundle of a manifold $M$ and CTM be its complexification. Let $S$ be a subbundle of $\mathrm{CTM}$ and suppose that $S \cap \bar{S}=\{0\}$, where $\bar{S}$ denotes the complex conjugate of $S$. Then there is a unique subbundle $P$ of $T M$ such that the complexification $C P$ of $P$ is written as $C P=S+\bar{S}$ (direct sum). $P$ is the real part of $S+\bar{S}$. Also we have a unique homomorphism $J: P \rightarrow P$, such that $J^{2}=-$ Id, where Id denotes the identity, and

$$
S=\{X-i J X ; X \in P\} \text {. }
$$

$\{P, J\}$ is called the real expression of $S$.

Furthermore, we suppose that $M$ is an $m$-dimensional contact manifold with a contact form $\eta$ and that the above $P$ is defined by $\eta=0$. If the form $L$ (the Levi form) defined by

$$
2 L(X, Y)=-d \eta(X, J Y), \quad X, Y \in P,
$$

is hermitian, then $(M, \eta, J)$ is called a nondegenerate, pseudohermitian manifold. This condition is equivalent to the partial integrability condition of $S$ :

$$
[\Gamma(S), \Gamma(S)] \subset \Gamma(\mathbf{C P}),
$$

where $\Gamma(S)$ denotes the space of all sections of $S$. If the integrability condition of $S$ :

$$
[\Gamma(S), \Gamma(S)] \subset \Gamma(S),
$$

is satisfied, then $(M, \eta, J)$ is said to be integrable.

If the Levi form $L$ is positive definite, then "nondegenerate" is replaced by "strongly pseudoconvex". A nondegenerate (strongly pseudoconvex, resp.), 
integrable pseudohermitian manifold is also called a nondegenerate (strongly pseudoconvex, resp.), integrable CR manifold.

The notion of strongly pseudoconvex, pseudohermitian structure is equivalent to the notion of contact Riemannian structure, by the relation $g=L+\eta \otimes \eta$, where the same letter $L$ denotes the natural extension to a $(0,2)$-tensor field on $M$.

We define a $(1,2)$-tensor field $Q$ on a contact Riemannian manifold by

$$
Q_{j k}^{i}=\nabla_{k} \phi_{j}^{i}+\xi^{i} \phi_{j}^{r} \nabla_{k} \eta_{r}+\phi_{r}^{i} \nabla_{k} \xi^{r} \eta_{j} \text {. }
$$

Proposition 2.1. Let $(M, \eta, g)$ be a contact Riemannian manifold. We define $J$ by $J=\phi \mid P$, where $P$ denotes the subbundle of $T M$ defined by $\eta=0$. Then $(M, \eta, J)$ is a strongly pseudoconvex, integrable $C R$ manifold, if and only if $Q=0$.

Proof. It suffices to show that the integrability condition

$$
[X-i \phi X, Y-i \phi Y] \in \Gamma(S), \quad X, Y \in \Gamma(P),
$$

is satisfied if and only if $Q=0$. Since $d \eta(X, Y)=d \eta(\phi X, \phi Y)$ holds on $(M, \eta, g)$, we see that $[X, Y]-[\phi X, \phi Y] \in \Gamma(P)$ (and $[\phi X, Y]+[X, \phi Y] \in$ $\Gamma(P))$ holds for $X, Y \in \Gamma(P)$. Therefore, (2.2) is equivalent to

$$
[\phi X, Y]+[X, \phi Y]=\phi[X, Y]-\phi[\phi X, \phi Y], \quad X, Y \in \Gamma(P),
$$

which is equivalent to

$$
\begin{aligned}
& \phi[\phi X, Y-\eta(Y) \xi]+\phi[X-\eta(X) \xi, \phi Y] \\
& \quad=-[X-\eta(X) \xi, Y-\eta(Y) \xi]+[\phi X, \phi Y], \quad X, Y \in \Gamma(T M) .
\end{aligned}
$$

That is,

$$
\begin{aligned}
\phi_{i}^{h} \nabla_{k} \phi_{h j}-\phi_{i}^{h} \nabla_{j} \phi_{h k}-\phi_{j}^{s} \nabla_{s} \phi_{i k}+\phi_{k}^{s} \nabla_{s} \phi_{i j} \\
-2 \eta_{i} \phi_{j k}+\eta_{j}\left(\nabla_{i} \eta_{k}+\nabla_{k} \eta_{i}\right)-\eta_{k}\left(\nabla_{i} \eta_{j}+\nabla_{j} \eta_{i}\right)=0 .
\end{aligned}
$$

Since $d \eta$ is closed, the third and the fourth terms of the left-hand side of (2.3) are calculated as follows:

$$
\begin{aligned}
-\phi_{j}^{s} \nabla_{s} \phi_{i k}+\phi_{k}^{s} \nabla_{s} \phi_{i j}= & \phi_{j}^{s}\left(\nabla_{i} \phi_{k s}+\nabla_{k} \phi_{s i}\right)-\phi_{k}^{s}\left(\nabla_{i} \phi_{j s}+\nabla_{j} \phi_{s i}\right) \\
= & \nabla_{i}\left(\eta_{j} \eta_{k}\right)+\nabla_{j}\left(\eta_{i} \eta_{k}\right)-\nabla_{k}\left(\eta_{i} \eta_{j}\right) \\
& +2 \phi_{k}^{s} \nabla_{i} \phi_{s j}-\phi_{i}^{s} \nabla_{k} \phi_{s j}+\phi_{i}^{s} \nabla_{j} \phi_{s k} .
\end{aligned}
$$

Therefore (2.3) is equivalent to

$$
\phi_{k}^{s} \nabla_{i} \phi_{s j}+\eta_{j} \nabla_{i} \eta_{k}=0
$$

which is equivalent to $Q=0$. Q.E.D.

Remark. If $m=2 n+1=3$, then $S$ is 1 -dimensional. So, the integrability condition (2.2) is a trivial consequence.

\section{The generalized Tanaka CONNection}

Tanaka [11] defined the canonical affine connection on a nondegenerate, integrable CR manifold. Generalizing this connection, we define the generalized 
Tanaka connection ${ }^{*} \nabla$ on a contact Riemannian manifold $(M, \eta, g)$ by

$$
{ }^{*} \Gamma_{j k}^{i}=\Gamma_{j k}^{i}+\eta_{j} \phi_{k}^{i}-\nabla_{j} \xi^{i} \eta_{k}+\xi^{i} \nabla_{j} \eta_{k},
$$

where the $\Gamma_{j k}^{i}$ denote the coefficients of the Riemannian connection $\nabla$. The torsion tensor ${ }^{*} T$ of ${ }^{*} \nabla$ is given by

$$
{ }^{*} T_{j k}^{i}=\eta_{j} \phi_{k}^{i}-\phi_{j}^{i} \eta_{k}-\nabla_{j} \xi^{i} \eta_{k}+\nabla_{k} \xi^{i} \eta_{j}+2 \xi^{i} \phi_{j k} .
$$

Proposition 3.1. The generalized Tanaka connection ${ }^{*} \nabla$ on a contact Riemannian manifold $(M, \eta, g)$ is a unique linear connection satisfying the following:

(i) ${ }^{*} \nabla \eta=0,{ }^{*} \nabla \xi=0$;

(ii) ${ }^{*} \nabla g=0$;

(iii-1) ${ }^{*} T(X, Y)=d \eta(X, Y) \xi, X, Y \in \Gamma(P)$;

(iii-2) ${ }^{*} T(\xi, \phi Y)=-\phi^{*} T(\xi, Y), Y \in \Gamma(P)$ or $Y \in \Gamma(T M)$;

(iv) ${ }^{*} \nabla_{X} \phi \cdot Y=Q(Y, X), X, Y \in \Gamma(T M)$.

Proof. (i), (ii), (iii-1) and (iv) are verified by (3.1) and (3.2). We show (iii-2). The left-hand side of (iii-2) is

$$
{ }^{*} T(\xi, \phi Y)=\phi \phi Y+\nabla_{\phi Y} \xi,
$$

where the second term of the last expression is calculated by using

$$
\begin{aligned}
\nabla_{r} \xi^{i} \phi_{j}^{r} & =\nabla_{r} \eta_{j} \phi^{r i}=\left(2 \phi_{r j}+\nabla_{j} \eta_{r}\right) \phi^{r i} \\
& =2 \delta_{j}^{i}-2 \xi^{i} \eta_{j}-\nabla_{j} \xi^{r} \phi_{r}^{i} .
\end{aligned}
$$

Thus (iii-2) is verified. Next, suppose that ${ }^{*} \nabla$ and ${ }^{*} \nabla{ }^{\prime}$ are connections satisfying (i)-(iv). Let $U$ be a $(1,2)$-tensor field which gives the difference between two connections. Then we can verify that $U$ vanishes. Q.E.D.

The Tanaka connection (in [11]) on a nondegenerate, integrable CR manifold is defined as a unique linear connection satisfying (i), (ii), (iii-1), (iii-2) and ${ }^{*} \nabla \phi=0$. So, our connection is a natural generalization. Notice that Proposition 3.1 works for nondegenerate, pseudohermitian manifolds.

Proposition 3.2. On a contact Riemannian manifold $(M, \eta, g)$,

$$
2\left\|^{*} T\right\|^{2}=\left\|L_{\xi} g\right\|^{2}+8(m-1)=\left\|L_{\xi} \phi\right\|^{2}+8(m-1)
$$

holds. In particular, $\left\|^{*} T\right\|^{2}$ attains its minimum $4(m-1)$ if and only if $\xi$ is a Killing vector field.

Proof. By (3.2) and $\phi^{i j} \nabla_{j} \eta_{i}=-(\phi ; \phi)=-(m-1)$ we obtain

$$
\left\|^{*} T\right\|^{2}=2\|\nabla \xi\|^{2}+2(m-1) .
$$

By $L_{\xi} g=\left(\nabla_{i} \eta_{j}+\nabla_{j} \eta_{i}\right)$ we obtain

$$
\left\|L_{\xi} g\right\|^{2}=2\|\nabla \xi\|^{2}+2 \nabla^{i} \xi^{j} \nabla_{j} \eta_{i} .
$$


Since $\nabla_{j} \eta_{i}=2 \phi_{j i}+\nabla_{i} \eta_{j}$ implies

$$
\nabla^{i} \xi^{j} \nabla_{j} \eta_{i}=\|\nabla \xi\|^{2}-2(m-1),
$$

we get

$$
\left\|L_{\xi} g\right\|^{2}=4\|\nabla \xi\|^{2}-4(m-1) \text {. }
$$

Since $L_{\xi} \phi_{j}^{i}=\phi^{i r} L_{\xi} g_{r j}$ and $\xi^{r} L_{\xi} g_{r j}=0$, it is easy to verify $\left\|L_{\xi} \phi\right\|^{2}=\left\|L_{\xi} g\right\|^{2}$. Q.E.D.

\section{The SPACE Of All asSociated RiemanNian METRICS}

Let $M$ be a contact manifold with a fixed contact form $\eta$. We denote the space of all Riemannian metrics associated with the contact form $\eta$ by $\mathscr{M}(\eta)$. We fix one of them and denote it by $g$. Let $\{g(t)\}$ be a curve in $\mathscr{M}(\eta)$ with $g(0)=g$. Then the structure tensors $\{\phi(t), \xi, \eta, g(t)\}$ corresponding to $g(t)$ satisfying the following:

$$
\begin{gathered}
g_{i r}(t) \xi^{r}=\eta_{i}, \\
2 g_{i r}(t) \phi_{j}^{r}(t)=2 \phi_{i j}=\nabla_{i} \eta_{j}-\nabla_{j} \eta_{i}, \\
\phi_{r}^{i}(t) \phi_{j}^{r}(t)=-\delta_{j}^{i}+\xi^{i} \eta_{j} .
\end{gathered}
$$

We put

$$
\begin{gathered}
g_{i j}(t)=g_{i j}+t h_{i j}+\left[t^{2}\right], \\
\phi_{j}^{i}(t)=\phi_{j}^{i}+t \varphi_{j}^{i}+\left[t^{2}\right],
\end{gathered}
$$

where $\left[t^{2}\right]$ denotes a set of the terms of higher order $(\geq 2)$ in $t$. Then $h$ and $\varphi$ satisfy the following:

$$
\begin{gathered}
h_{i r} \xi^{r}=0, \quad \eta_{r} \varphi_{j}^{r}=0, \quad \varphi_{r}^{i} \xi^{r}=0, \\
h_{i r} \phi_{j}^{r}+g_{i r} \varphi_{j}^{r}=0, \\
\varphi_{r}^{i} \phi_{j}^{r}+\phi_{r}^{i} \varphi_{j}^{r}=0 .
\end{gathered}
$$

By (4.6)-(4.8) we can verify that $h$ satisfies

$$
h_{i j}+h_{r s} \phi_{i}^{r} \phi_{j}^{s}=0 \text {, i.e. }, 2 h_{i j}=h_{i j}-h_{r s} \phi_{i}^{r} \phi_{j}^{s} \text {, }
$$

and $\varphi$ is expressed as

$$
\varphi_{j}^{i}=-h_{r}^{i} \phi_{j}^{r}
$$

By (4.6) and (4.9) we see that $h$ is expressed as

$$
h=\left(\begin{array}{ccc}
0 & 0 & 0 \\
0 & B & C \\
0 & C & -B
\end{array}\right)
$$


with respect to an adapted frame at a point of $M$, where $B$ and $C$ are symmetric $n \times n$ matrices.

Here (and in the following) we use the following notation:

(i) For $h=\left(h_{i j}\right), h^{+}$means $\left(h_{j}^{i}\right)$;

(ii) For $S=\left(S_{i j}\right)$ and $T=\left(T_{j}^{i}\right), S \cdot T$ means $\left(S_{i r} T_{j}^{r}\right)$.

Proposition 4.1. Let $\{g(t)\}$ be a smooth curve in $\mathscr{M}(\eta)$ such that $g(0)=g$. Then $h$ in (4.4) satisfies

$$
h^{+} \xi=0, \quad h^{+} \phi=-\phi h^{+} .
$$

Conversely, let $h$ be an arbitrary symmetric $(0,2)$-tensor field on $M$ satisfying (4.12). Then $\{g(t)\}$, defined by

$$
g(t)=g \cdot e^{t h^{+}}, \quad-\varepsilon<t<\varepsilon,
$$

is a smooth curve in $\mathscr{M}(\eta)$ such that $g(0)=g$.

Proof. It is easy to see that the two conditions $h_{i r} \xi^{r}=0$ and (4.9) are equivalent to (4.12). So, we prove the second half. By the definition of $g(t)$ we get $g(t)(X, Y)=g\left(X, e^{t h^{+}} Y\right), X, Y \in \Gamma(T M)$. We define $\phi(t)$ by $\phi(t)=\phi e^{t h^{+}}$. Then (4.12) gives

$$
e^{t h^{+}} \phi=\phi e^{-t h^{+}} \text {. }
$$

Thus, we can verify three relations (4.1)-(4.3), and hence $\phi(t), \xi, \eta$ and $g(t)$ are structure tensors corresponding to $g(t)$. Q.E.D.

Remark. (i) For a smooth curve $\{g(t)\}$ in $\mathscr{M}(\eta)$, the volume element $d M(t)$ is equal to $d M$. This corresponds to the fact that $\eta \wedge(d \eta)^{n}$ is unchanged.

(ii) Let $A=\left(A_{j}^{i}\right)$ be an arbitrary $(1,1)$-tensor field on a contact Riemannian manifold $(M, \eta, g)$ such that $A \xi=0$ and $A_{i j}=g_{i r} A_{j}^{r}$ is symmetric. Then $h$ defined by $h^{+}=A+\phi A \phi$ satisfies (4.12).

\section{The Dirichlet ENERGY $E(g)$}

Let $M$ be a compact contact manifold with a fixed contact form $\eta$. We notice here that a contact manifold is orientable. For each $g \in \mathscr{M}(\eta)$ we associate the generalized Tanaka connection ${ }^{*} \nabla$ and we consider the Dirichlet energy $\int_{M}\left\|^{*} T\right\|^{2} d M$. By Proposition 3.2 (cf. also Proposition 6.1 below), it is equivalent to consider the following

$$
E(g)=\int_{M}\left\|L_{\xi} g\right\|^{2} d M .
$$

This Dirichlet energy was first studied by Chern-Hamilton [3] in the case of CR manifolds of dimension 3. Since this integral has a natural meaning also in contact Riemannian manifolds of general odd dimensions, we study critical metrics $g$ of $(5.1)$ with respect to deformations in $\mathscr{M}(\eta)$. 
Let $\{g(t)\}$ be a curve in $\mathscr{M}(\eta)$ with $g(0)=g$. We follow the notations used in $\S 4$, and put $g(t)$ and $\phi(t)$ as (4.4) and (4.5). Now we calculate the integrand of $E(t)=E(g(t))$.

$$
\begin{aligned}
\left\|L_{\xi} g(t)\right\|^{2} & =\left(g^{i r}-t h^{i r}\right)\left(g^{j s}-t h^{j s}\right)\left(L_{\xi} g_{i j}+t L_{\xi} h_{i j}\right)\left(L_{\xi} g_{r s}+t L_{\xi} h_{r s}\right)+\left[t^{2}\right] \\
& =\left\|L_{\xi} g\right\|^{2}-2 t\left[h^{i r} g^{j s} L_{\xi} g_{i j} L_{\xi} g_{r s}-\left(L_{\xi} h ; L_{\xi} g\right)\right]+\left[t^{2}\right] .
\end{aligned}
$$

We show $h^{i r} g^{j s} L_{\xi} g_{i j} L_{\xi} g_{r s}=0$. By (4.9) we get

$$
2 h^{i r} g^{j s} L_{\xi} g_{i j} L_{\xi} g_{r s}=\left(h^{i r}-h^{a b} \phi_{a}^{i} \phi_{b}^{r}\right)\left(\nabla_{i} \eta_{j}+\nabla_{j} \eta_{i}\right)\left(\nabla_{r} \xi^{j}+\nabla^{j} \eta_{r}\right)
$$

which vanishes by Lemma 1.1. Therefore we obtain

$$
\frac{d E}{d t}(0)=\frac{d}{d t}\left(\int_{M}\left\|L_{\xi} g(t)\right\|^{2} d M(t)\right)(0)=2 \int_{M}\left(L_{\xi} h ; L_{\xi} g\right) d M .
$$

By Stokes-Green's theorem we obtain

$$
\begin{aligned}
\int_{M}\left(L_{\xi} h ; L_{\xi} g\right) d M & =\int_{M}\left(\xi^{k} \nabla_{k} h_{i j}+\nabla_{i} \xi^{k} h_{k j}+\nabla_{j} \xi^{k} h_{i k}\right)\left(\nabla^{i} \xi^{j}+\nabla^{j} \xi^{i}\right) d M \\
= & \int_{M}\left[-h_{i j} \xi^{k} \nabla_{k}\left(\nabla^{i} \xi^{j}+\nabla^{j} \xi^{i}\right)\right. \\
& \left.\quad+\left(\nabla_{i} \xi^{k} h_{k j}+\nabla_{j} \xi^{k} h_{i k}\right)\left(\nabla^{i} \xi^{j}+\nabla^{j} \xi^{i}\right)\right] d M \\
= & \int_{M} h_{i j}\left[-\xi^{k} \nabla_{k}\left(\nabla^{i} \xi^{j}+\nabla^{j} \xi^{i}\right)+2 \nabla_{r} \xi^{i}\left(\nabla^{r} \xi^{j}+\nabla^{j} \xi^{r}\right)\right] d M .
\end{aligned}
$$

By Lemma 1.1 and (4.9) we obtain

$$
\begin{aligned}
2 h_{i j}\left[-\xi^{k} \nabla_{k}\left(\nabla^{i} \xi^{j}+\nabla^{j} \xi^{i}\right)\right] & =-\left(h_{i j}-h_{a b} \phi_{i}^{a} \phi_{j}^{b}\right) \xi^{k} \nabla_{k}\left(\nabla^{i} \xi^{j}+\nabla^{j} \xi^{i}\right) \\
& =-2 h_{i j} \xi^{k} \nabla_{k}\left(\nabla^{i} \xi^{j}+\nabla^{j} \xi^{i}\right), \\
2 h_{i j} \nabla_{r} \xi^{i}\left(\nabla^{r} \xi^{j}+\nabla^{j} \xi^{r}\right) & \\
= & \left(h_{i j}-h_{a b} \phi_{i}^{a} \phi_{j}^{b}\right) \nabla_{r} \xi^{i}\left(\nabla^{r} \xi^{j}+\nabla^{j} \xi^{r}\right) \\
& =h_{i j} \nabla_{r} \xi^{i}\left(\nabla^{r} \xi^{j}+\nabla^{j} \xi^{r}\right)+h_{a b} \phi_{r}^{i} \nabla^{a} \eta_{i} \cdot \phi_{j}^{r}\left(\nabla^{b} \xi^{j}+\nabla^{j} \xi^{b}\right) \\
& =h_{j}^{i} \nabla_{r} \eta_{i}\left(\nabla^{r} \xi^{j}+\nabla^{j} \xi^{r}\right)-h_{b}^{a} \nabla_{a} \eta_{i}\left(\nabla^{b} \xi^{i}+\nabla^{i} \xi^{b}\right) \\
& =2 h_{j}^{i} \phi_{r i}\left(\nabla^{r} \xi^{j}+\nabla^{j} \xi^{r}\right) .
\end{aligned}
$$

Therefore, summarizing the above we obtain

$$
\frac{d E}{d t}(0)=-2 \int_{M}\left(h ; \nabla_{\xi} L_{\xi} g-2 L_{\xi} g \cdot \phi\right) d M .
$$

Now we prove the following.

Theorem 5.1. Let $(M, \eta)$ be a compact contact manifold. Then an associated metric $g \in \mathscr{M}(\eta)$ is critical with respect to (5.1) if and only if

$$
\nabla_{\xi} L_{\xi} g=2 L_{\xi} g \cdot \phi .
$$


Proof. Assume that $E$ is critical at $g$. We define $h$ by

$$
h=\nabla_{\xi} L_{\xi} g-2 L_{\xi} g \cdot \phi \text {. }
$$

Then $h$ is symmetric and $h^{+} \xi=0$ holds. Furthermore, we can verify that $h^{+} \phi=-\phi h^{+}$holds. By Proposition 4.1, we see that $g(t)=g \cdot e^{t h^{+}}$defines a smooth curve in $\mathscr{M}(\eta)$. We apply (5.2) to this deformation to get $h=0$. Q.E.D. $E$.

$g \in \mathscr{M}(\eta)$ is said to be $E$-critical if $g$ is a critical point of the functional

Remark. The condition (5.3) of our Theorem 5.1 is different from the condition (for $m=3$ ) obtained by Chern-Hamilton [3, p. 299]. This is because [3, p . $289,(36) \ldots$ ] contain unhappy small errors.

Remark. Blair [2] proved the following: Let $(M, \eta, g)$ be a regular compact contact Riemannian manifold. Then $g$ is $E$-critical if and only if $\xi$ is a Killing vector field.

Proposition 5.2. $g$ is E-critical if and only if

$$
\eta_{s} R_{i r j}^{s} \xi^{r}=2 g_{i j}-2 \eta_{i} \eta_{j}-\nabla_{r} \eta_{i} \nabla^{r} \eta_{j}
$$

Proof. The left-hand side of (5.3) is written as

$$
\begin{aligned}
\xi^{r} \nabla_{r}\left(L_{\xi} g_{i j}\right) & =\xi^{r} \nabla_{r}\left(2 \phi_{j i}+2 \nabla_{i} \eta_{j}\right) \\
& =2 \xi^{r} \nabla_{r} \nabla_{i} \eta_{j} \\
& =2 \xi^{r} R_{j i r}^{s} \eta_{s}-2 \nabla_{i} \xi^{r} \nabla_{r} \eta_{j},
\end{aligned}
$$

where we used the Ricci identity. On the other hand,

$$
\begin{aligned}
2 \phi_{i}^{r}\left(L_{\xi} g_{r j}\right) & =2 \phi_{i}^{r}\left(2 \phi_{j r}+2 \nabla_{r} \eta_{j}\right) \\
& =-4\left(g_{i j}-\eta_{i} \eta_{j}\right)+2\left(\nabla^{r} \eta_{i}-\nabla_{i} \xi^{r}\right) \nabla_{r} \eta_{j} .
\end{aligned}
$$

Thus, we obtain (5.4).

Corollary 5.3. Suppose that $g$ is E-critical. If $\left\|\nabla_{X} \xi\right\|=\left\|\nabla_{Y} \xi\right\|$ holds for unit vectors $X, Y$ in $P$, then the sectional curvatures satisfy $K(\xi, \phi X)=K(\xi, \phi Y)$.

Proof. By (5.4), it suffices to notice the following:

$$
\nabla_{r} \eta_{i} \nabla^{r} \eta_{j} \phi_{a}^{i} \phi_{b}^{j}=\nabla_{a} \eta_{i} \nabla_{b} \eta_{j} \phi_{r}^{i} \phi^{j r}=\nabla_{a} \eta_{i} \nabla_{b} \xi^{i}
$$

\section{THE GENERALIZED WEBSTER TORSION}

Let $(M, \eta, g)$ be a contact Riemannian manifold. Let $\left\{\xi, e_{\alpha}, e_{\bar{\alpha}}\right\}$ be an adapted frame field (locally defined). The dual frame to $\left\{\xi, e_{\alpha}, e_{\bar{\alpha}}\right\}$ is denoted by $\left\{\eta, w^{\alpha}, w^{\bar{\alpha}}\right\}$. In this section we show that the Webster torsion $\tau^{\alpha}$ can be 
also defined for $(M, \eta, g)$. First we notice that the torsion tensor ${ }^{*} T$ of the generalized Tanaka connection is written as

$$
{ }^{*} T_{j k}^{i}=\frac{1}{2} g^{i r}\left(L_{\xi} g_{r k} \eta_{j}-L_{\xi} g_{r j} \eta_{k}\right)+2 \xi^{i} \phi_{j k} .
$$

Then we see that the components of ${ }^{*} T$ with respect to an adapted frame $\left\{\xi, e_{\alpha}, e_{\bar{\alpha}}\right\}$ are given by

$$
\begin{gathered}
{ }^{*} T_{0 \beta}^{\alpha}=-{ }^{*} T_{\beta 0}^{\alpha}=-{ }^{*} T_{0 \bar{\beta}}^{\bar{\alpha}}={ }^{*} T_{\bar{\beta} 0}^{\bar{\alpha}}=\frac{1}{2}\left(L_{\xi} g\right)\left(e_{\alpha}, e_{\beta}\right), \\
{ }^{*} T_{0 \bar{\beta}}^{\alpha}=-{ }^{*} T_{\bar{\beta} 0}^{\alpha}={ }^{*} T_{0 \beta}^{\bar{\alpha}}=-{ }^{*} T_{\beta 0}^{\bar{\alpha}}=\frac{1}{2}\left(L_{\xi} g\right)\left(e_{\alpha}, e_{\bar{\beta}}\right), \\
{ }^{*} T_{\alpha \bar{\beta}}^{0}=-{ }^{*} T_{\bar{\beta} \alpha}^{0}=-2 \delta_{\alpha \beta}, \\
\text { the others }=0 .
\end{gathered}
$$

Now we put

$$
\begin{gathered}
\theta^{\alpha}=\frac{1}{\sqrt{2}}\left(w^{\alpha}+i w^{\bar{\alpha}}\right), \quad i=\sqrt{-1}, \\
E_{\alpha}=\frac{1}{\sqrt{2}}\left(e_{\alpha}-i e_{\bar{\alpha}}\right),
\end{gathered}
$$

and $\theta^{\bar{\alpha}}=\overline{\theta^{\alpha}}, E_{\bar{\alpha}}=\overline{E_{\alpha}}$. Then the torsion of a nondegenerate, integrable pseudohermitian structure defined by Webster [18] is

$$
\tau^{\alpha}=\left(d \theta^{\alpha}\left(\xi, E_{\bar{\beta}}\right)\right) \theta^{\bar{\beta}}
$$

This is well defined also in a contact Riemannian manifold. Let $\left(w_{k}^{j}\right)$ be the connection form of $\nabla$ with respect to $\left\{\xi, e_{\alpha}, e_{\bar{\alpha}}\right\}$. Then $d \theta^{\alpha}\left(\xi, E_{\bar{\beta}}\right)$ is given by

$$
\begin{aligned}
2 d \theta^{\alpha}\left(\xi, E_{\bar{\beta}}\right)= & -w_{\beta}^{\alpha}(\xi)+w_{\bar{\beta}}^{\bar{\alpha}}(\xi)+w_{0}^{\alpha}\left(e_{\beta}\right)-w_{0}^{\bar{\alpha}}\left(e_{\bar{\beta}}\right) \\
& +i\left\{-w_{\beta}^{\bar{\alpha}}(\xi)-w_{\bar{\beta}}^{\alpha}(\xi)+w_{0}^{\bar{\alpha}}\left(e_{\beta}\right)+w_{0}^{\alpha}\left(e_{\bar{\beta}}\right)\right\} .
\end{aligned}
$$

By $w_{k}^{j}(X)=w^{j}\left(\nabla_{X} e_{k}\right), \nabla_{\xi} \phi=0$ and $\nabla_{i} \eta_{j}=\phi_{i j}+(1 / 2) L_{\xi} g_{i j}$, we get

$$
2 \tau^{\alpha}=\left\{\left(L_{\xi} g\right)\left(e_{\alpha}, e_{\beta}\right)+i\left(L_{\xi} g\right)\left(e_{\alpha}, e_{\beta}\right)\right\} \theta^{\bar{\beta}} \text {. }
$$

Thus, we get the following.

Proposition 6.1. In a contact Riemannian manifold $(M, \eta, g)$, the relation between the generalized Webster torsion $\tau=\left(\tau^{\alpha}\right)$ and ${ }^{*} T$ is given by

$$
\tau^{\alpha}(X)=\theta^{\alpha}\left[{ }^{*} T(\xi, X)\right], \quad X \in \Gamma(\mathbf{C} T M),
$$

and the norm of $\tau$ is given by

$$
\|\tau\|^{2}=2 \sum_{\alpha=1}^{n}\left\|\tau^{\alpha}\right\|^{2}=\frac{1}{2}\left\|L_{\xi} g\right\|^{2} .
$$

Remark. Since the language of adapted moving frames for the complexified tangent bundle, as in Webster [18], is useful also in the case of contact Riemannian 
manifolds, we give the structure equations with respect to $\left\{\xi, E_{\alpha}, E_{\bar{\alpha}}\right\}$. First we get $\phi E_{\alpha}=i E_{\alpha}, g\left(E_{\alpha}, E_{\bar{\beta}}\right)=\delta_{\alpha \beta}$ and

$$
d \eta=-2 i \theta^{\alpha} \wedge \theta^{\bar{\alpha}} \text {. }
$$

By $\left(\omega_{k}^{j}\right)$ we denote the connection form of $\nabla$ with respect to $\left\{\xi, E_{\alpha}, E_{\bar{\alpha}}\right\}$. Then $\nabla_{\xi} \eta=0$ and $\nabla_{\xi} \xi=0$. imply $\omega_{k}^{0}(\xi)=\omega_{0}^{j}(\xi)=0$. By $\nabla_{\xi} \phi=0$, we get $\omega_{\bar{\beta}}^{\alpha}(\xi)=\omega_{\beta}^{\bar{\alpha}}(\xi)=0$. Hence, we can put

$$
\begin{gathered}
\omega_{0}^{\alpha}=B_{\beta}^{\alpha} \theta^{\beta}+A_{\bar{\beta}}^{\alpha} \theta^{\bar{\beta}}=-\omega_{\bar{\alpha}}^{0}, \\
\omega_{\bar{\beta}}^{\alpha}=C_{\bar{\beta} \gamma}^{\alpha} \theta^{\gamma}+C_{\bar{\beta} \bar{\gamma}}^{\alpha} \theta^{\bar{\gamma}} .
\end{gathered}
$$

Here, we can verify that $B_{\beta}^{\alpha}=-i \delta_{\beta}^{\alpha} \cdot A_{\bar{\beta}}^{\alpha}$ is determined by (6.1). We use the notation $A_{\bar{\alpha} \bar{\beta}}=A_{\bar{\beta}}^{\alpha}$ and $A_{\alpha \beta}=\overline{A_{\bar{\alpha} \bar{\beta}}}$. Then we can verify that $4 A_{\alpha \beta} \theta^{\alpha} \otimes \theta^{\beta}=$ $L_{\xi} g-i L_{\xi} g \cdot \phi$. Now, our connection ${ }^{*} \nabla$ is expressed by ${ }^{*} \omega_{k}^{0}={ }^{*} \omega_{0}^{j}=0$ and

$$
d \theta^{\alpha}=\theta^{\beta} \wedge^{*} \omega_{\beta}^{\alpha}+\theta^{\bar{\beta}} \wedge^{*} \omega_{\bar{\beta}}^{\alpha}+\eta \wedge \tau^{\alpha},
$$

where ${ }^{*} \omega_{\beta}^{\alpha}=\omega_{\beta}^{\alpha}+i \delta_{\beta}^{\alpha} \eta,{ }^{*} \omega_{\bar{\beta}}^{\alpha}=\omega_{\bar{\beta}}^{\alpha}$ and $\tau^{\alpha}=A_{\bar{\beta}}^{\alpha} \theta^{\bar{\beta}}$.

The referee kindly pointed out that Theorem 5.1 can be easily proved by using (6.3). In this case, we notice that (5.3) is equivalent to ${ }^{*} \nabla_{\xi} A_{\alpha \beta}=0$.

\section{A CONDITION CONCERNING ${ }^{*} T$}

Concerning the torsion tensor ${ }^{*} T$, we consider one condition $\nabla_{\xi}{ }^{*} T=0$, because it has some geometric meaning. First, we prepare a lemma.

Lemma 7.1. In a contact Riemannian manifold, sectional curvatures $K(\xi, X)$ and $K(\xi, \phi X)$ satisfy the following.

$$
K(\xi, X)-K(\xi, \phi X)=-\left(\nabla_{\xi} L_{\xi} g\right)(X, X)
$$

for a unit vector $X$ in $P$.

Proof. By the Ricci identity and Lemma 1.1 we get

$$
2 R_{i r j s} \xi^{r} \xi^{s}=-2 \nabla_{j} \eta_{s} \nabla^{s} \eta_{i}-\xi^{s} \nabla_{s}\left(\nabla_{j} \eta_{i}+\nabla_{i} \eta_{j}\right) .
$$

Operating $\phi_{a}^{i} \phi_{b}^{j}$ to (7.1) and using Lemma 1.1, we obtain

$$
\begin{aligned}
2 R_{i r j s} \xi^{r} \xi^{s} \phi_{a}^{i} \phi_{b}^{j} & =-2 \nabla_{j} \eta_{s} \phi_{b}^{j} \nabla^{s} \eta_{i} \phi_{a}^{i}-\phi_{a}^{i} \phi_{b}^{j} \xi^{s} \nabla_{s}\left(\nabla_{j} \eta_{i}+\nabla_{i} \eta_{j}\right) \\
& =-2 \nabla_{j} \eta_{b} \phi_{s}^{j} \nabla^{s} \eta_{i} \phi_{a}^{i}+\xi^{s} \nabla_{s}\left(\nabla_{b} \eta_{a}+\nabla_{a} \eta_{b}\right) \\
& =-2 \nabla_{j} \eta_{b} \nabla_{a} \xi^{j}+\xi^{s} \nabla_{s}\left(\nabla_{b} \eta_{a}+\nabla_{a} \eta_{b}\right) .
\end{aligned}
$$

Therefore we obtain

$$
R_{i r j s} \xi^{r} \xi^{s}-R_{a r b s} \xi^{r} \xi^{s} \phi_{i}^{a} \phi_{j}^{b}=-\xi^{s} \nabla_{s}\left(L_{\xi} g_{i j}\right),
$$

which completes the proof. 
Proposition 7.2. In a contact Riemannian manifold, the following five conditions are equivalent:

(i) $\nabla_{\xi}^{*} T=0$;

(ii) $\nabla_{\xi} \nabla \xi=0$;

(iii) $\nabla_{\xi} L_{\xi} g=0$;

(iv) $K(\xi, X)=K(\xi, \phi X), X \in \Gamma(P),\|X\|=1$;

(v) $K(\xi, X)=K(\xi, \sin \theta X+\cos \theta \phi X), X \in \Gamma(P),\|X\|=1,0 \leq \theta<\pi$.

Proof. Assume that $\nabla_{\xi}{ }^{*} T=0$. Then applying Lemma 1.1 we obtain

$$
0=\xi^{s} \nabla_{s}\left(\phi_{k}^{i} \eta_{j}-\phi_{j}^{i} \eta_{k}-\nabla_{j} \xi^{i} \eta_{k}+\nabla_{k} \xi^{i} \eta_{j}\right)=\xi^{s} \nabla_{s} \nabla_{k} \xi^{i} \eta_{j}-\xi^{s} \nabla_{s} \nabla_{j} \xi^{i} \eta_{k}
$$

Since $\xi^{s} \nabla_{s} \nabla_{j} \xi^{i} \xi^{j}=-\xi^{s} \nabla_{j} \xi^{i} \nabla_{s} \xi^{j}=0$, we get $\xi^{s} \nabla_{s} \nabla_{k} \xi^{i}=0$. So, (i) and (ii) are equivalent. Since

$$
\nabla_{i} \eta_{j}+\nabla_{j} \eta_{i}=2 \nabla_{i} \eta_{j}+2 \phi_{j i}
$$

and $\nabla_{\xi} \phi=0$, we see that (ii) and (iii) are equivalent. By Lemma 7.1, (iii) and (iv) are equivalent. It is easy to show that (iv) and (v) are equivalent. Q.E.D.

Proposition 7.3. Let $(M, \eta, g)$ be a contact Riemannian manifold with $\nabla_{\xi}{ }^{*} T$ $=0$.

(i) Let $X$ be a unit parallel vector field along a trajectory (which is geodesic) of $\xi$ such that $g(\xi, X)=0$. Then the sectional curvature $K(\xi, X)$ is constant along the trajectory.

(ii) Ricci curvature $\operatorname{Ric}(\xi, \xi)$ is constant along each trajectory of $\xi$, and is equal to $2(m-1)-\|\nabla \xi\|^{2}$.

Proof. $\nabla_{\xi} \nabla \xi=0$ and (7.1) imply

$$
\xi^{k} \nabla_{k} R_{i r j s} \xi^{r} \xi^{s}=0 .
$$

Let $X$ be one satisfying the condition of (i). Then (7.3) implies

$$
\xi^{k} \nabla_{k}\left(R_{i r j s} \xi^{r} \xi^{s} X^{i} X^{j}\right)=0,
$$

which proves (i). Contracting (7.3) with $g^{i j}$, we get $\xi^{k} \nabla_{k} R_{r s} \xi^{r} \xi^{s}=0$. This proves the first part of (ii). The second part follows from (3.4) and (7.1). Q.E.D.

Corollary 7.4. Let $(M, \eta, g)$ be a 3-dimensional contact Riemannian manifold with $\nabla_{\xi}{ }^{*} T=0$. Then, for each point $x$ of $M$, the sectional curvature $K(\xi, X)$ is independent of the choice of $X$ in $P$ at $x$. If we denote this value by $k(x)$, then $k$ is constant along each trajectory of $\xi$.

\section{The generalized TANAKa-Webster scalar CURVature}

We calculate the curvature tensor of ${ }^{*} \nabla$. Let $W$ be the $(1,2)$-tensor field expressing the difference between ${ }^{*} \nabla$ and $\nabla$ :

$$
W_{j k}^{i}={ }^{*} \Gamma_{j k}^{i}-\Gamma_{j k}^{i}
$$


Then

$$
{ }^{*} R_{j k l}^{i}=R_{j k l}^{i}+\nabla_{k} W_{l j}^{i}-\nabla_{l} W_{k j}^{i}+W_{l j}^{s} W_{k s}^{i}-W_{k j}^{s} W_{l s}^{i} .
$$

Therefore we obtain

$$
\begin{aligned}
{ }^{*} R_{j k l}^{i}= & R_{j k l}^{i}+\nabla_{k} \phi_{j}^{i} \eta_{l}-\nabla_{l} \phi_{j}^{i} \eta_{k}+2 \phi_{j}^{i} \phi_{k l} \\
& -\phi_{r}^{i} \nabla_{l} \xi^{r} \eta_{j} \eta_{k}+\phi_{r}^{i} \nabla_{k} \xi^{r} \eta_{j} \eta_{l}+\xi^{i} \nabla_{k} \eta_{r} \phi_{j}^{r} \eta_{l}-\xi^{i} \nabla_{l} \eta_{r} \phi_{j}^{r} \eta_{k} \\
& -R_{s k l}^{i} \xi^{s} \eta_{j}-\xi^{i} R_{j k l}^{s} \eta_{s}+\nabla_{k} \xi^{i} \nabla_{l} \eta_{j}-\nabla_{l} \xi^{i} \nabla_{k} \eta_{j} .
\end{aligned}
$$

Contracting the above with respect to $i$ and $k$, we obtain

$$
{ }^{*} R_{j l}=R_{j l}+2 g_{j l}-2 \eta_{j} \eta_{l}-\eta_{j} R_{s l} \xi^{s}-R_{r j s l} \xi^{r} \xi^{s}-\nabla_{r} \eta_{j} \nabla_{l} \xi^{r} \text {. }
$$

Since ${ }^{*} R_{j l} \xi^{j} \xi^{l}=0$, we define the generalized Tanaka-Webster scalar curvature ${ }^{*} S$ for a contact Riemannian manifold $(M, \eta, g)$ by ${ }^{*} S=g^{j l *} R_{j l}$ (cf. Tanaka [11, p. 186], Webster [18]). By (7.1) and (3.4), we obtain

$$
\nabla_{r} \eta_{s} \nabla^{s} \xi^{r}=-R_{r s} \xi^{r} \xi^{s}=-2(m-1)+\|\nabla \xi\|^{2}
$$

Thus, we obtain

$$
{ }^{*} S=S-R_{r s} \xi^{r} \xi^{s}+4 n
$$

where $S$ denotes the scalar curvature of $(M, g)$.

\section{GAUGE TRANSFORMATIONS OF CONTACT RiEMANNIAN STRUCTURE}

Let $(M, \eta, g)$ be a contact Riemannian manifold and let $\sigma$ be a positive function on $M$. We consider a new contact form $\tilde{\eta}=\sigma \eta$ and define structure tensors $(\tilde{\phi}, \tilde{\xi}, \tilde{g})$ corresponding to $\tilde{\eta}$ under one condition:

(*) For each point $x$ of $M$, the actions of $\phi$ and $\tilde{\phi}$ are identical for $P_{x}$ (i.e., the subspace of $T_{x} M$ defined by $\eta=0$ ).

By calculating $d \tilde{\eta}=d(\sigma \eta)$, we obtain

$$
2 \tilde{\phi}_{i j}=\sigma_{i} \eta_{j}-\sigma_{j} \eta_{i}+2 \sigma \phi_{i j}
$$

where $\sigma_{i}=\nabla_{i} \sigma$. By $\tilde{\xi}^{i} \tilde{\phi}_{i j}=0, \tilde{\eta}_{i} \tilde{\xi}^{i}=1$ and $(9.1)$, we obtain $\tilde{\xi} \sigma=(1 / \sigma) \xi \sigma$, and

$$
\tilde{\xi}^{k}=\frac{1}{\sigma} \xi^{k}+\frac{1}{2 \sigma^{2}} \phi_{j}^{k} \sigma^{j}
$$

So we define $\zeta$ by $\zeta^{k}=(1 / 2 \sigma) \phi_{j}^{k} \sigma^{j}$ and get

$$
\tilde{\xi}^{k}=\frac{1}{\sigma}\left(\xi^{k}+\zeta^{k}\right)
$$

By $\tilde{\phi}_{i j} \tilde{\phi}^{j k}=-\delta_{i}^{k}+\tilde{\xi}^{k} \tilde{\eta}_{i}$ and $\tilde{\eta}_{j} \tilde{\phi}^{j k}=0, \tilde{\phi}^{j k}$ is determined:

$$
\tilde{\phi}^{j k}=\frac{1}{\sigma} \phi^{j k} .
$$


Now, by condition (*) we can put $\tilde{\phi}_{j}{ }^{i}=\phi_{j}^{i}+v^{i} \eta_{j}$ for some vector field $v$ on $M$. By $\tilde{\eta}_{i} \tilde{\phi}_{j}{ }^{i}=0$ and $\tilde{\phi}_{j}{ }^{i} \tilde{\phi}_{k}^{j}=-\delta_{k}^{i}+\tilde{\xi}^{i} \tilde{\eta}_{k}, v$ is determined:

$$
v^{i}=\frac{1}{2 \sigma}\left(\sigma^{i}-\xi \sigma \cdot \xi^{i}\right)
$$

By the expressions of $\tilde{\phi}_{i j}$ and $\tilde{\phi}_{j}^{i}$, we obtain

$$
\tilde{g}_{i j}=\sigma\left(g_{i j}-\eta_{i} \zeta_{j}-\zeta_{i} \eta_{j}\right)+\sigma\left(\sigma-1+\|\zeta\|^{2}\right) \eta_{i} \eta_{j}
$$

The inverse matrix $\left(\tilde{g}^{j k}\right)$ of $\left(\tilde{g}_{i j}\right)$ is given by

$$
\tilde{g}^{j k}=\frac{1}{\sigma}\left(g^{j k}-\xi^{j} \xi^{k}\right)+\frac{1}{\sigma^{2}}\left(\xi^{j}+\zeta^{j}\right)\left(\xi^{k}+\zeta^{k}\right) .
$$

The last relation is rewritten as

$$
\sigma\left(\tilde{g}^{j k}-\tilde{\xi}^{j} \tilde{\xi}^{k}\right)=g^{j k}-\xi^{j} \xi^{k} .
$$

Summarizing the above we obtain the following.

Lemma 9.1. Under condition (*), a gauge transformation $\eta \rightarrow \tilde{\eta}=\sigma \eta$ of $a$ contact form $\eta$ induces the transformation of the structure tensors of the following form:

$$
\begin{gathered}
\tilde{\xi}^{i}=\frac{1}{\sigma}\left(\xi^{i}+\zeta^{i}\right), \quad \zeta^{i}=\frac{1}{2 \sigma} \phi_{j}^{i} \sigma^{j}, \\
\tilde{\phi}_{j}^{i}=\phi_{j}^{i}+\frac{1}{2 \sigma}\left(\sigma^{i}-\xi \sigma \cdot \xi^{i}\right) \eta_{j}, \\
\tilde{g}_{i j}=\sigma\left(g_{i j}-\eta_{i} \zeta_{j}-\zeta_{i} \eta_{j}\right)+\sigma\left(\sigma-1+\|\zeta\|^{2}\right) \eta_{i} \eta_{j} .
\end{gathered}
$$

We call the transformation of the structure tensors given by Lemma 9.1 a gauge transformation of contact Riemannian structure.

10. The generalized TANAKA-Webster scalar CURVATURe UNDER GAUGE TRANSFORMATIONS

Let $f$ and $f^{\prime}$ be two functions on a contact Riemannian manifold $(M, \eta, g)$. We define an operator $\Delta_{P}$ acting on the space of functions by using the Laplacian $\Delta$ and $\xi$ :

$$
\Delta_{P} f=\Delta f-\xi \xi f=\left(g^{i j}-\xi^{i} \xi^{j}\right) \nabla_{i} \nabla_{j} f,
$$

and $\left(d f ; d f^{\prime}\right)_{P}$ by

$$
\left(d f ; d f^{\prime}\right)_{P}=\left(g^{i j}-\xi^{i} \xi^{j}\right) \nabla_{i} f \nabla_{j} f^{\prime}
$$

Furthermore, $\|d f\|_{P}^{2}$ means $(d f ; d f)_{P}$, which is equal to $\|d f\|^{2}-(\xi f)^{2}$. 
Theorem 10.1. Let $(\eta, g) \rightarrow(\tilde{\eta}=\sigma \eta, \tilde{g})$ be a gauge transformation of contact Riemannian structure. Then the transformation of the generalized TanakaWebster scalar curvature ${ }^{*} S$ is given by

$$
\sigma^{*} \tilde{S}={ }^{*} S-\frac{m+1}{\sigma} \Delta_{P} \sigma-\frac{(m+1)(m-5)}{4 \sigma^{2}}\|d \sigma\|_{P}^{2} .
$$

Proof. Since $\tilde{g}_{i j}$ in Lemma 9.1 is complicated, the direct calculation is somewhat difficult. So, we give here a method of better calculation, showing some essential steps. Geometric quantities corresponding to $\tilde{g}$ are denoted by $\sim$. We define $\widetilde{W}_{j k}^{i}$ by

Then

$$
\widetilde{W}_{j k}^{i}=\widetilde{\Gamma}_{j k}^{i}-\Gamma_{j k}^{i}
$$

$$
\widetilde{W}_{j k}^{i}=\frac{1}{2} \tilde{g}^{i a}\left(\nabla_{j} \tilde{g}_{a k}+\nabla_{k} \tilde{g}_{a j}-\nabla_{a} \tilde{g}_{j k}\right) \text {. }
$$

The Ricci tensor $\widetilde{R}_{j l}$ is given by

$$
\widetilde{R}_{j l}=R_{j l}+\nabla_{r} \widetilde{W}_{l j}^{r}-\nabla_{l} \widetilde{W}_{r j}^{r}+\widetilde{W}_{l j}^{s} \widetilde{W}_{r s}^{r}-\widetilde{W}_{r j}^{s} \widetilde{W}_{l s}^{r} .
$$

Transvecting the last equality by $g^{j l}-\xi^{j} \xi^{l}$ and using (9.4) we obtain

$$
\begin{aligned}
\sigma\left(\widetilde{S}-\widetilde{R}_{j l} \tilde{\xi}^{j} \tilde{\xi}^{l}\right)= & S-R_{j l} \xi^{j} \xi^{l}+\left(g^{j l}-\xi^{j} \xi^{l}\right) \nabla_{r} \widetilde{W}_{l j}^{r} \\
& -\left(g^{j l}-\xi^{j} \xi^{l}\right) \nabla_{l} \widetilde{W}_{r j}^{r}+\left(g^{j l}-\xi^{j} \xi^{l}\right) \widetilde{W}_{l j}^{s} \widetilde{W}_{r s}^{r} \\
& -\left(g^{j l}-\xi^{j} \xi^{l}\right) \widetilde{W}_{r j}^{s} \widetilde{W}_{l s}^{r} .
\end{aligned}
$$

Step 1. The third term of the right-hand side of $(10.2)$ is

$$
\left(g^{j l}-\xi^{j} \xi^{l}\right) \nabla_{r} \widetilde{W}_{l j}^{r}=\nabla_{r}\left(\widetilde{W}_{l j}^{r}\left(g^{j l}-\xi^{j} \xi^{l}\right)\right)+\nabla_{r}\left(\xi^{j} \xi^{l}\right) \widetilde{W}_{l j}^{r} .
$$

First we calculate the following:

$$
\begin{aligned}
\widetilde{W}_{l j}^{r}\left(g^{j l}-\xi^{j} \xi^{l}\right)= & \frac{1}{2} \tilde{g}^{r a}\left(\nabla_{l} \tilde{g}_{a j}+\nabla_{j} \tilde{g}_{a l}-\nabla_{a} \tilde{g}_{j l}\right) \sigma\left(\tilde{g}^{j l}-\tilde{\xi}^{j} \tilde{\xi}^{l}\right) \\
= & \sigma \tilde{g}^{r a} \nabla_{j} \tilde{g}_{a l}\left(\tilde{g}^{j l}-\tilde{\xi}^{j} \tilde{\xi}^{l}\right)-\frac{1}{2} \sigma \tilde{g}^{r a} \nabla_{a} \tilde{g}_{j l}\left(\tilde{g}^{j l}-\tilde{\xi}^{j} \tilde{\xi}^{l}\right) \\
= & \sigma \tilde{g}^{r a}\left[\nabla_{j}\left(-\tilde{\xi}^{j} \tilde{\eta}_{a}\right)-\nabla_{j}\left(\frac{1}{\sigma}\left(g^{j l}-\xi^{j} \xi^{l}\right)\right) \tilde{g}_{a l}\right] \\
& -\frac{1}{2} \sigma \tilde{g}^{r a} \tilde{g}_{j l} \nabla_{a}\left(\frac{1}{\sigma}\left(g^{j l}-\xi^{j} \eta^{l}\right)\right) .
\end{aligned}
$$

After calculation we obtain

$$
\widetilde{W}_{l j}^{r}\left(g^{j l}-\xi^{j} \xi^{l}\right)=\frac{m-1}{2 \sigma} \xi \sigma \cdot \xi^{r}-\frac{m-1}{2 \sigma} \sigma^{r}
$$

and

$$
\nabla_{r}\left(\widetilde{W}_{l j}^{r}\left(g^{j l}-\xi^{j} \xi^{l}\right)\right)=\frac{m-1}{2 \sigma^{2}}\|d \sigma\|_{P}^{2}-\frac{m-1}{2 \sigma} \Delta_{P} \sigma .
$$

Next, calculating

$$
\nabla_{r}\left(\xi^{j} \xi^{l}\right) \widetilde{W}_{l j}^{r}=\nabla_{r} \xi^{j} \xi^{l} \tilde{g}^{r a}\left(\nabla_{l} \tilde{g}_{a j}+\nabla_{j} \tilde{g}_{a l}-\nabla_{a} \tilde{g}_{j l}\right),
$$


we obtain

(10.5)

$$
\begin{aligned}
\nabla_{r}\left(\xi^{j} \xi^{l}\right) \widetilde{W}_{l j}^{r}= & \frac{1}{\sigma}\left(\sigma^{r} \zeta^{s}+\sigma^{s} \zeta^{r}\right) \nabla_{s} \eta_{r}+\frac{1}{\sigma^{2}} \xi \sigma \cdot \zeta^{r} \zeta^{s} \nabla_{s} \eta_{r} \\
& -2(m-1)\left(\sigma-1+\|\zeta\|^{2}\right)+\frac{2}{\sigma} \zeta^{r} \nabla_{r} \eta_{j} \zeta^{a} \nabla^{j} \eta_{a}+2 \phi_{j r} \nabla^{j} \zeta^{r} \\
& +\frac{1}{\sigma} \zeta^{r} \nabla_{r} \eta_{j}\left(\nabla^{j} \zeta_{a}+\nabla_{a} \zeta^{j}\right) \zeta^{a}+\frac{1}{\sigma^{2}}\left(1-\|\zeta\|^{2}\right) \sigma^{j} \zeta^{r} \nabla_{r} \eta_{j}
\end{aligned}
$$

Step 2. The fourth term of the right-hand side of (10.2) is

$$
-\left(g^{j l}-\xi^{j} \xi^{l}\right) \nabla_{l} \widetilde{W}_{r j}^{r}=-\frac{1}{2}\left(g^{j l}-\xi^{j} \xi^{l}\right) \nabla_{l}\left(\tilde{g}^{r a} \nabla_{j} \tilde{g}_{r a}\right) .
$$

By a direct calculation we get

$$
\tilde{g}^{r a} \nabla_{j} \tilde{g}_{r a}=\frac{m+1}{\sigma} \sigma_{j}
$$

Therefore

$$
-\left(g^{j l}-\xi^{j} \xi^{l}\right) \nabla, \widetilde{W}_{r j}^{r}=-\frac{m+1}{2 \sigma} \Delta_{P} \sigma+\frac{m+1}{2 \sigma^{2}}\|d \sigma\|_{P}^{2}
$$

Step 3. The fifth term of the right-hand side of (10.2) is

$$
\left(g^{j l}-\xi^{j} \xi^{l}\right) \widetilde{W}_{l j}^{s} \widetilde{W}_{r s}^{r}=-\frac{(m+1)(m-1)}{4 \sigma^{2}}\|d \sigma\|_{P}^{2}
$$

by (10.3) and (10.6).

Step 4. The sixth term of the right-hand side of (10.2) is

$$
\begin{aligned}
& -\left(g^{j l}-\xi^{j} \xi^{l}\right) \widetilde{W}_{r j}^{s} \widetilde{W}_{l s}^{r} \\
& \quad=\frac{1}{4}\left(g^{j l}-\xi^{j} \xi^{l}\right)\left[\nabla_{j} \tilde{g}_{r s} \nabla_{l} \tilde{g}^{r s}+2 \nabla_{a} \tilde{g}_{j r} \nabla_{s} \tilde{g}_{l b} \tilde{g}^{s a} \tilde{g}^{r b}-2 \nabla_{a} \tilde{g}_{j r} \nabla_{b} \tilde{g}_{l s} \tilde{g}^{s a} \tilde{g}^{r b}\right] .
\end{aligned}
$$

The right-hand side of the last equality is calculated as follows:

$$
\begin{aligned}
\frac{1}{4}\left(g^{j l}\right. & \left.-\xi^{j} \xi^{l}\right) \nabla_{j} \tilde{g}_{r s} \nabla_{l} \tilde{g}^{r s} \\
= & -\frac{m+3}{4 \sigma^{2}}\|d \sigma\|_{P}^{2}+\frac{1}{2}\left(2-\sigma-\frac{1}{\sigma}-\|\zeta\|^{2}\right)\|\nabla \xi\|^{2} \\
& +\left(1-\frac{1}{\sigma}\right)(\nabla \xi ; \nabla \zeta)+\frac{1}{\sigma} \xi^{r} \sigma^{s} \nabla_{s} \zeta_{r}-\frac{1}{2 \sigma}\|\nabla \zeta\|^{2}+\frac{1}{\sigma} \zeta^{r} \nabla_{l} \zeta_{r} \eta_{s} \nabla^{l} \zeta^{s} \\
& -\frac{1}{2 \sigma}\left(\sigma+1+\|\zeta\|^{2}\right) \xi^{r} \nabla_{l} \zeta_{r} \eta_{s} \nabla^{l} \zeta^{s}+\frac{1}{2 \sigma}\left\|\nabla_{\xi} \zeta\right\|^{2},
\end{aligned}
$$




$$
\begin{aligned}
\frac{1}{2}\left(g^{j l}\right. & \left.-\xi^{j} \xi^{l}\right) \nabla_{a} \tilde{g}_{j r} \nabla_{s} \tilde{g}_{l b} \tilde{g}^{s a} \tilde{g}^{r b} \\
= & \frac{m-1}{2 \sigma^{2}}\left[\|d \sigma\|^{2}+\left(\frac{1}{\sigma}-1\right)(\xi \sigma)^{2}\right] \\
& +\left(\frac{1}{2 \sigma}(\sigma-1)^{2}+\|\zeta\|^{2}\right)\|\nabla \xi\|^{2}+\frac{1}{\sigma}(1-\sigma)(\nabla \xi ; \nabla \zeta) \\
& +\left[\frac{1}{2 \sigma^{2}}(\sigma-1)^{2}+\frac{1}{\sigma}\|\zeta\|^{2}\right]\left\|\nabla_{\zeta} \xi\right\|^{2}+\frac{1}{2 \sigma}\|\nabla \zeta\|^{2} \\
& +\frac{1}{\sigma^{2}}(1-\sigma) \zeta^{a} \nabla_{a} \eta_{r}\left(\xi^{b}+\zeta^{b}\right) \nabla_{b} \zeta^{r}-\frac{2}{\sigma} \zeta^{r} \sigma^{s} \nabla_{s} \eta_{r}-\frac{2}{\sigma^{2}} \xi \sigma \cdot \zeta^{r} \zeta^{s} \nabla_{s} \eta_{r} \\
& +\frac{1}{\sigma} \zeta^{s} \nabla_{a} \eta_{s} \zeta^{l} \nabla^{a} \zeta_{l}+\frac{1}{2 \sigma}\left(1+\|\zeta\|^{2}\right) \zeta^{r} \nabla_{a} \eta_{r} \zeta^{s} \nabla^{a} \eta_{s} \\
& +\frac{1}{2 \sigma^{2}}(1-\sigma)\left\|\nabla_{\xi} \zeta\right\|^{2}+\frac{1}{2 \sigma^{2}}\left(2 \xi^{a}+\zeta^{a}\right) \nabla_{a} \zeta_{l} \zeta^{b} \nabla_{b} \zeta^{l} \\
& +\frac{1}{\sigma^{2}} \zeta^{s} \zeta^{a} \nabla_{a} \eta_{s} \zeta^{l}\left(\xi^{b}+\zeta^{b}\right) \nabla_{b} \zeta_{l}+\frac{1}{2 \sigma^{2}}\left(1+\|\zeta\|^{2}\right)\left(\zeta^{r} \zeta^{s} \nabla_{s} \eta_{r}\right)^{2} \\
-\frac{1}{2}\left(g^{j l}\right. & \left.-\xi^{j} \xi^{l}\right) \nabla_{a} \tilde{g}_{j r} \nabla_{b} \tilde{g}_{l s} \tilde{g}^{s a} \tilde{g}^{r b} \\
= & -\frac{1}{2 \sigma^{2}}\|d \sigma\|_{P}^{2}+\frac{1}{\sigma} \sigma^{r} \zeta^{s} \nabla_{s} \eta_{r} \\
& +\frac{1}{\sigma^{2}} \xi \sigma\left(\nabla_{r} \zeta^{r}+\zeta^{r} \zeta^{s} \nabla_{s} \eta_{r}\right)-\frac{1}{2}\|\zeta\|^{2} \nabla_{b} \eta_{a} \nabla^{a} \xi^{b}+\frac{1}{\sigma}\left(\nabla_{\zeta} \xi ; \nabla_{\xi} \zeta\right) \\
& -\frac{1}{\sigma}\left(1+\|\zeta\|^{2}\right) \zeta^{b} \nabla_{b} \eta_{a} \zeta^{s} \nabla^{a} \eta_{s}-\frac{1}{\sigma} \zeta^{b} \nabla_{b} \eta_{a} \zeta^{r} \nabla^{a} \zeta_{r} \\
& -\frac{1}{2 \sigma^{2}}\left\|\nabla_{\zeta} \xi+\nabla_{\xi} \zeta+\nabla_{\zeta} \zeta\right\|^{2} \\
& -\frac{1}{2 \sigma^{2}}\left(1+\|\zeta\|^{2}\right)\left(\zeta^{r} \zeta^{s} \nabla_{s} \eta_{r}\right)^{2}-\frac{1}{\sigma^{2}} \zeta^{r} \zeta^{a} \nabla_{a} \eta_{r}\left(\xi^{b}+\zeta^{b}\right) \zeta^{s} \nabla_{b} \zeta_{s} .
\end{aligned}
$$

Since $\nabla_{r} \zeta^{r}=-(m-1) \xi \sigma / 2 \sigma$, we obtain

$$
\begin{aligned}
-\left(g^{j l}-\xi^{j} \xi^{l}\right) \widetilde{W}_{r j}^{s} \widetilde{W}_{l s}^{r}=\frac{m-3}{4 \sigma^{2}}\|d \sigma\|_{P}^{2}+\|\zeta\|^{2} \phi_{s r} \nabla^{s} \xi^{r} \\
-\frac{1}{2} \zeta^{r} \nabla_{l} \eta_{r} \zeta^{s} \nabla^{l} \eta_{s}-\frac{1}{\sigma^{2}} \xi \sigma \cdot \zeta^{r} \zeta^{s} \nabla_{s} \eta_{r}-\frac{2}{\sigma} \zeta^{r} \sigma^{s} \nabla_{s} \eta_{r} \\
\quad-\frac{1}{\sigma}\left(1+\|\zeta\|^{2}\right) \zeta^{b} \nabla_{b} \eta_{r} \zeta^{s} \nabla^{r} \eta_{s}-\frac{1}{\sigma} \zeta^{a} \nabla_{a} \eta_{r}\left(\nabla_{b} \zeta^{r}+\nabla^{r} \zeta_{b}\right) \zeta^{b} \\
\quad+\left(\frac{1}{2}-\frac{1}{\sigma}+\frac{1}{\sigma}\|\zeta\|^{2}\right)\left\|\nabla_{\zeta} \xi\right\|^{2} .
\end{aligned}
$$

Step 5. Since

$$
\begin{aligned}
\left(\nabla_{a} \eta_{r} \nabla_{b} \xi^{r}-\nabla_{r} \eta_{a} \nabla^{r} \eta_{b}\right) \zeta^{a} \zeta^{b} & =-2\left(\phi_{a}^{r} \nabla_{b} \eta_{r}+\phi_{a}^{r} \nabla_{r} \eta_{b}\right) \zeta^{a} \zeta^{b} \\
& =\frac{1}{\sigma}\left(\nabla_{b} \eta_{a}+\nabla_{a} \eta_{b}\right) \sigma^{a} \zeta^{b}
\end{aligned}
$$


etc., summarizing (10.4), (10.5), (10.7)-(10.9), we get

$$
\begin{aligned}
\sigma\left(\widetilde{S}-\widetilde{R}_{j l} \tilde{\xi}^{j} \tilde{\xi}^{l}\right)= & S-R_{j l} \xi^{j} \xi^{l}-2(m-1)(\sigma-1) \\
& -\frac{m+1}{\sigma} \Delta_{P} \sigma-\frac{(m+1)(m-5)}{4 \sigma^{2}}\|d \sigma\|_{P}^{2},
\end{aligned}
$$

from which we obtain (10.1). Q.E.D.

If we define $u$ and $\mu$ by

$$
\sigma=u^{2 / n}, \quad \mu=\frac{4(n+1)}{n},
$$

then (10.1) is written as

$$
-\mu \Delta_{P} u+{ }^{*} S u={ }^{*} \widetilde{S} u^{(n+2) / n} .
$$

This corresponds to the identity for a strongly pseudoconvex, integrable CR manifold by Jerison-Lee [4].

Suppose that some constant ${ }^{*} \widetilde{S}$ is given. Then solving $u$ satisfying $(10.10)$ on a contact Riemannian manifold is a problem corresponding to the Yamabe problem in conformal geometry.

\section{A gaUge INVARIANT OF CONTACT RIEMANNIAN STRUCTURE}

In this section we obtain an invariant by gauge transformations of contact Riemannian structure. This is a generalization of an invariant for strongly pseudoconvex, integrable CR structure obtained by Jerison-Lee [4].

Let $(M, \eta, g)$ be a compact contact Riemannian manifold. We consider $\eta \rightarrow \tilde{\eta}=\sigma \eta$ and follow the notations in $\S 10$.

Lemma 11.1. For a function $f$ on $M$,

$$
\tilde{\Delta}_{P} f=\frac{1}{\sigma} \Delta_{P} f+\frac{n}{\sigma^{2}}(d \sigma ; d f)_{P} .
$$

Proof. By definition of $\Delta_{P}$ and $\tilde{\Delta}_{P}$ we obtain

$$
\begin{aligned}
\tilde{\Delta}_{P} f & =\left(\tilde{g}^{r s}-\tilde{\xi}^{r} \tilde{\xi}^{s}\right) \tilde{\nabla}_{r} f_{s} \\
& =\frac{1}{\sigma}\left(g^{r s}-\xi^{r} \xi^{s}\right)\left(\nabla_{r} f_{s}-\widetilde{W}_{r s}^{a} f_{a}\right) \\
& =\frac{1}{\sigma} \Delta_{P} f-\frac{1}{\sigma}\left(g^{r s}-\xi^{r} \xi^{s}\right) \widetilde{W}_{r s}^{a} f_{a} .
\end{aligned}
$$

Applying (10.3) to the last line, we get (11.1). Q.E.D.

We put $\sigma=u^{2 / n}$ as before and $p=2+2 / n$. Then $\widetilde{d M}=u^{p} d M$ follows. For a nonnegative function $f$ on $M$, we define $\tilde{f}$ by $\tilde{f}=f / u$. Then

$$
\int_{M} \tilde{f}^{p} \widetilde{d M}=\int_{M} f^{p} d M
$$

Lemma 11.2. The following equality holds:

$$
-\mu \tilde{\Delta}_{P} \tilde{f}+{ }^{*} \tilde{S} \tilde{f}=u^{1-p}\left(-\mu \Delta_{P} f+{ }^{*} S f\right) .
$$


Proof. Calculation is straightforward by using (11.1) for $\tilde{\Delta}_{P}$ and (10.10) for ${ }^{*} \tilde{S}$. Q.E.D.

By (11.3) we see that

$$
\int_{M}\left(-\mu \tilde{\Delta}_{P} \tilde{f}+{ }^{*} \tilde{S} \tilde{f}\right) \tilde{f} \widetilde{d M}=\int_{M}\left(-\mu \Delta_{P} f+{ }^{*} S f\right) f d M
$$

holds for any nonnegative function $f$ on $M$. Since

$$
\int_{M} \Delta_{P} f \cdot f d M=-\int_{M}\|d f\|_{P}^{2} d M
$$

we define $\kappa_{(\eta, g)}$ by

$$
\kappa_{(\eta, g)}=\inf \left\{\int_{M}\left(\mu\|d f\|_{P}^{2}+{ }^{*} S f^{2}\right) d M ; f \geq 0, \int_{M} f^{p} d M=1\right\} .
$$

Then (11.2), (11.4) and (11.5) imply the following.

Theorem 11.3. Let $(M, \eta, g)$ be a compact contact Riemannian manifold. Then $\kappa_{(\eta, g)}$ is invariant under gauge transformations of contact Riemannian structure. Remark. For the related results on strongly pseudo-convex, integrable CR manifolds, see Jerison-Lee $[4,5,6]$.

Theorem 11.3 shows some importance of the integral given in (11.6). We study this integral in the following sections.

\section{THE FIRST VARIATION OF THE FUNCTIONAL $F_{(\eta)}$}

Let $(M, \eta)$ be a compact contact manifold. By $\mathscr{F}(p)$ we denote the space of all functions on $(M, \eta)$ satisfying $f \geq 0$ and $\int_{M} f^{p} d M=1$, and we consider the following functional:

$$
\begin{gathered}
F_{(\eta)}: \mathscr{M}(\eta) \times \mathscr{F}(p) \rightarrow \mathbf{R}, \\
F_{(\eta)}(g, f)=\int_{M}\left(\mu\|d f\|_{P}^{2}+{ }^{*} S f^{2}\right) d M .
\end{gathered}
$$

As a special case, fixing $g$, we consider the functional: $F_{(\eta, g)}: \mathscr{F}(p) \rightarrow \mathbf{R}$, defined by $F_{(\eta, g)}(f)=F_{(\eta)}(g, f)$.

Let $\{g(t)\}$ and $\{f(t)\}$ be smooth curves in $\mathscr{M}(\eta)$ and $\mathscr{F}(p)$ such that $g(0)=g$ and $f(0)=f$, respectively. We calculate $d F(t) / d t$ at $t=0$, where $F(t)=F_{(\eta)}(g(t), f(t))$ :

$$
\begin{aligned}
F(t)=\int_{M}\left[\mu\left(g^{i j}(t)-\xi^{i} \xi^{j}\right) \nabla_{i} f(t) \nabla_{j} f(t)\right. \\
\left.+\left(S(t)-R_{i j}(t) \xi^{i} \xi^{j}+4 n\right) f(t)^{2}\right] d M .
\end{aligned}
$$

We put

$$
\begin{gathered}
g_{i j}(t)=g_{i j}+t h_{i j}+\left[t^{2}\right], \\
f(t)=f+t \psi+\left[t^{2}\right] .
\end{gathered}
$$


Then $\int_{M} f(t)^{p} d M=1$ implies

$$
\int_{M} f^{p-1} \psi d M=0 \text {. }
$$

$h_{r}^{r}=0$ and (12.2) imply (cf. (13.3) and (13.4) below)

$$
\begin{gathered}
g^{i j}(t)=g^{i j}-t h^{i j}+\left[t^{2}\right], \\
S(t)=S+t\left(\nabla^{r} \nabla^{s} h_{r s}-h^{r s} R_{r s}\right)+\left[t^{2}\right], \\
R_{i j}(t)=R_{i j}+\frac{t}{2}\left(\nabla_{r} \nabla_{i} h_{j}^{r}+\nabla_{r} \nabla_{j} h_{i}^{r}-\nabla_{r} \nabla^{r} h_{i j}\right)+\left[t^{2}\right] .
\end{gathered}
$$

Noticing that $h_{r s} \xi^{r}=0$, we get

$$
-\left(\nabla_{r} \nabla_{i} h_{j}^{r}+\nabla_{r} \nabla_{j} h_{i}^{r}-\nabla_{r} \nabla^{r} h_{i j}\right) \xi^{i} \xi^{j}=4 h^{i j} \nabla_{r} \eta_{i} \phi_{j}^{r}+2 \nabla_{i} h_{j}^{r} \xi^{i} \nabla_{r} \xi^{j},
$$

and hence we obtain

$$
\frac{d F}{d t}(0)=2 \int_{M}\left(-\mu \Delta_{P} f+{ }^{*} S f\right) \psi d M+\int_{M}(h ; N) d M,
$$

where, putting $b=2-\mu=-2(n+2) / n, N=\left(N_{i j}\right)$ is defined by

$$
N_{i j}=2 f \nabla_{i} \nabla_{j} f+b \nabla_{i} f \nabla_{j} f-f^{2} R_{i j}+2 f^{2} \nabla_{r} \eta_{i} \phi_{j}^{r}-\frac{1}{2} \xi^{r} \nabla_{r}\left(f^{2} L_{\xi} g_{i j}\right) .
$$

Since $h_{i r} \xi^{r}=0$, we obtain $(h ; N)=\left(h ; N^{\prime}\right)$, where

$$
\begin{aligned}
N_{i j}^{\prime}= & 2 f \nabla_{i} \nabla_{j} f-2 f \xi^{r}\left(\eta_{i} \nabla_{r} \nabla_{j} f+\eta_{j} \nabla_{r} \nabla_{i} f\right)+2 f \xi \xi f \cdot \eta_{i} \eta_{j} \\
& +b \nabla_{i} f \nabla_{j} f-b \xi f\left(\eta_{i} \nabla_{j} f+\eta_{j} \nabla_{i} f\right)+b(\xi f)^{2} \eta_{i} \eta_{j} \\
& -f^{2}\left(R_{i j}-R_{i r} \xi^{r} \eta_{j}-R_{j r} \xi^{r} \eta_{i}+R_{r s} \xi^{r} \xi^{s} \eta_{i} \eta_{j}\right) \\
& +2 f^{2} \nabla_{r} \eta_{i} \phi_{j}^{r}-\frac{1}{2} \xi^{r} \nabla_{r}\left(f^{2} L_{\xi} g_{i j}\right) .
\end{aligned}
$$

By (4.9) we get $\left(h ; N^{\prime}\right)=(h ; H)$, where $2 H_{i j}=\left(\delta_{i}^{r} \delta_{j}^{s}-\phi_{i}^{r} \phi_{j}^{s}\right) N_{r s}^{\prime}$, i.e., (12.6)

$$
\begin{aligned}
2 H_{i j}= & 2 f\left(\nabla_{i} \nabla_{j} f-\nabla_{r} \nabla_{s} f \phi_{i}^{r} \phi_{j}^{s}\right)-2 f \xi^{r}\left(\eta_{i} \nabla_{r} \nabla_{j} f+\eta_{j} \nabla_{r} \nabla_{i} f\right)+2 f \xi \xi f \cdot \eta_{i} \eta_{j} \\
& +b\left(\nabla_{i} f \nabla_{j} f-\nabla_{r} f \nabla_{s} f \phi_{i}^{r} \phi_{j}^{s}\right)-b \xi f\left(\eta_{i} \nabla_{j} f+\eta_{j} \nabla_{i} f\right)+b(\xi f)^{2} \eta_{i} \eta_{j} \\
& -f^{2}\left(R_{i j}-R_{r r} \phi_{i}^{r} \phi_{j}^{s}-R_{i r} \xi^{r} \eta_{j}-R_{j r} \xi^{r} \eta_{i}+R_{r s} \xi^{r} \xi^{s} \eta_{i} \eta_{j}\right) \\
& +2 f^{2} L_{\xi} g_{i r} \phi_{j}^{r}-\xi^{r} \nabla_{r}\left(f^{2} L_{\xi} g_{i j}\right),
\end{aligned}
$$

where we have used $L_{\xi} g_{r s} \phi_{i}^{r} \phi_{j}^{s}=-L_{\xi} g_{i j}$, etc. (12.5) is now

$$
\frac{d F}{d t}(0)=2 \int_{M}\left(-\mu \Delta_{P} f+{ }^{*} S f\right) \psi d M+\int_{M}(h ; H) d M .
$$

Proposition 12.1. For a compact contact Riemannian manifold $(M, \eta, g)$, the functional $F_{(\eta)}$ is critical at the pair $(g, f)$, if and only if $g$ and $f$ satisfy

$$
\begin{gathered}
-\mu \Delta_{P} f+{ }^{*} S f=c f^{p-1}, \\
H=0,
\end{gathered}
$$

where $c$ is a constant and $H=\left(H_{i j}\right)$ is defined by (12.6). 
Proof. Suppose that $g$ and $f$ satisfy (12.8) and (12.9). Then (12.7) implies that $d F(0) / d t=0$ holds for any $\psi$ satisfying (12.4) and for any $h$ coming from $\{g(t)\}$. Conversely, suppose that $F_{(\eta)}$ is critical at the pair $(g, f)$. We define a constant $c$ by

$$
c=\int_{M}\left(-\mu \Delta_{P} f+{ }^{*} S f\right) f^{p-1} d M / \int_{M} f^{2 p-2} d M
$$

and a function $\psi$ by

$$
\psi=-\mu \Delta_{P} f+{ }^{*} S f-c f^{p-1} .
$$

Then $\int_{M} f^{p-1} \psi d M=0$ holds. Let $\{f(t)\}$ be a curve in $\mathscr{F}(p)$ to which $\psi$ is tangent. We define $h$ by $h=H$, where $H$ is defined by (12.6). Let $\{g(t)\}$ be a curve in $\mathscr{M}(\eta)$ to which $h$ is tangent. This is possible by Proposition 4.1, since $h$ satisfies (4.12). Then (12.7) gives

$$
\frac{d F}{d t}(0)=2 \int_{M} \psi \psi d M+\int_{M}(h ; h) d M .
$$

Hence we obtain $\psi=0$ and $H=0$. Q.E.D.

Remark. For a standard contact Riemannian structure $\left(\eta, g_{0}\right)$ on the unit sphere $\left(S^{m}, g_{0}\right)$ with $m=2 n+1, F_{(\eta)}$ is critical at the pair $\left(g_{0}, f_{0}\right)$, where $f_{0}=\left(\operatorname{vol}\left(S^{m}, g_{0}\right)\right)^{-1 / p}$. In this case, ${ }^{*} S=4 n(n+1)$ and $c=4 n(n+1) f_{0}^{2-p}$.

Proposition 12.2. Let $(M, \eta, g)$ be a compact contact Riemannian manifold with constant ${ }^{*} S$. Then the functional $F_{(\eta)}$ is critical at the pair $(g, f)$ with $f=(\operatorname{vol}(M, g))^{-1 / p}$, if and only if

$$
\begin{gathered}
R_{i j}-R_{r s} \phi_{i}^{r} \phi_{j}^{s}-R_{i r} \xi^{r} \eta_{j}-R_{j r} \xi^{r} \eta_{i}+R_{r s} \xi^{r} \xi^{s} \eta_{i} \eta_{j} \\
=2 L_{\xi} g_{i r} \phi_{j}^{r}-\xi^{r} \nabla_{r}\left(L_{\xi} g_{i j}\right) .
\end{gathered}
$$

Proof. Since $f$ is constant, the condition $H_{i j}=0$ in $(12.6)$ is equivalent to (12.10). Q.E.D.

Corollary 12.3. Let $(M, \eta, g)$ be a compact contact Einstein Riemannian manifold such that $\xi$ is a Killing vector field. Then $F_{(\eta)}$ is critical at the pair $(g, f)$ with $f=(\operatorname{vol}(M, g))^{-1 / p}$.

Proposition 12.4. Let $(M, \eta, g)$ be a compact contact Riemannian manifold. Then $g$ is a critical point of the functional $\int_{M}{ }^{*} S d M$ on $\mathscr{M}(\eta)$, if and only if (12.10) holds.

Proof. In the above discussion, if one puts $f(t)=$ constant in (12.1), then the proof is complete.

Remark. If $m=3$, the condition (12.10) is equivalent to the fact that $\xi$ is a Killing vector field. In fact, let $X$ be a unit vector in $P$. Transvecting (12.10) with $X^{i} \phi_{r}^{j} X^{r}$ and using (7.2), we get

$$
\operatorname{Ric}(X, \phi X)-g(X, R(\phi X, \xi) \xi)=-\left(L_{\xi} g\right)(X, X),
$$


where Ric denotes the Ricci tensor. Putting $X=e_{1}$, etc., we see that $L_{\xi} g=0$ holds. To prove the converse, assume that $\xi$ is a Killing vector field. Then, considering a fibering $U \rightarrow U / \xi$ of an open set $U$ of $(M, \eta, g)$ and noticing that the dimension of $U / \xi$ is two, we see that the Ricci tensor is of the form Ric $=a g+b \eta \otimes \eta($ cf. $[12$, p. 313]). So, both sides of (12.10) vanish.

\section{THE SECOND DERIVATIVE OF $F(t)$}

Let $(M, g)$ be a Riemannian manifold. For a 1-parameter family $\{g(t)\}$ of Riemannian metrics such that $g(0)=g$, we put

$$
g_{i j}(t)=g_{i j}+t h_{i j}+t^{2} k_{i j}+\left[t^{3}\right],
$$

where $\left(h_{i j}\right)$ and $\left(k_{i j}\right)$ are symmetric. The inverse $\left(g^{i j}(t)\right)$ of $\left(g_{i j}(t)\right)$ is given by

$$
g^{i j}(t)=g^{i j}-t h^{i j}+t^{2}\left(h_{r}^{i} h^{r j}-k^{i j}\right)+\left[t^{3}\right],
$$

where $k^{i j}=g^{i r} g^{j s} k_{r s}$, etc. We define $W_{j k}^{i}(t)$ by

$$
W_{j k}^{i}(t)=\Gamma_{j k}^{i}(t)-\Gamma_{j k}^{i}
$$

Then

$$
\begin{aligned}
W_{j k}^{i}(t)= & \frac{t}{2}\left(\nabla_{j} h_{k}^{i}+\nabla_{k} h_{j}^{i}-\nabla^{i} h_{j k}\right) \\
& +\frac{t^{2}}{2}\left[\left(\nabla_{j} k_{k}^{i}+\nabla_{k} k_{j}^{i}-\nabla^{i} k_{j k}\right)-h^{i r}\left(\nabla_{j} h_{r k}+\nabla_{k} h_{r j}-\nabla_{r} h_{j k}\right)\right]+\left[t^{3}\right] .
\end{aligned}
$$

Calculating

$$
R_{j l}(t)=R_{j l}+\nabla_{r} W_{l j}^{r}(t)-\nabla_{l} W_{r j}^{r}(t)+W_{l j}^{s}(t) W_{r s}^{r}(t)-W_{r j}^{s}(t) W_{l s}^{r}(t)+\left[t^{3}\right],
$$

and $g^{j l}(t) R_{l j}(t)$, we obtain

$$
\begin{aligned}
& R_{j l}(t)=R_{j l}+\frac{t}{2}\left(\nabla_{r} \nabla_{l} h_{j}^{r}+\nabla_{r} \nabla_{j} h_{l}^{r}-\nabla_{r} \nabla^{r} h_{j l}-\nabla_{l} \nabla_{j} h_{r}^{r}\right) \\
& +\frac{t^{2}}{4}\left[2 \nabla_{r} \nabla_{l} k_{j}^{r}+2 \nabla_{r} \nabla_{j} k_{l}^{r}-2 \nabla_{r} \nabla^{r} k_{j l}-2 \nabla_{l} \nabla_{j} k_{r}^{r}\right. \\
& \quad-2 h^{r s}\left(\nabla_{r} \nabla_{l} h_{s j}+\nabla_{r} \nabla_{j} h_{s l}-\nabla_{r} \nabla_{s} h_{j l}-\nabla_{l} \nabla_{j} h_{r s}\right) \\
& \quad-2 \nabla_{s} h^{s r}\left(\nabla_{l} h_{r j}+\nabla_{j} h_{r l}-\nabla_{r} h_{j l}\right)+\nabla_{l} h^{r s} \nabla_{j} h_{r s} \\
& +\nabla_{r} h_{s}^{s}\left(\nabla_{l} h_{j}^{r}+\nabla_{j} h_{l}^{r}-\nabla^{r} h_{j l}\right) \\
& \left.+2 \nabla_{r} h_{s j} \nabla^{r} h_{l}^{s}-2 \nabla_{r} h_{s j} \nabla^{s} h_{l}^{r}\right]+\left[t^{3}\right],
\end{aligned}
$$




$$
\begin{aligned}
& S(t)=S+t\left(\nabla^{r} \nabla^{s} h_{r s}-\nabla_{r} \nabla^{r} h_{s}^{s}-h^{r s} R_{r s}\right) \\
& +t^{2}\left[h_{r}^{i} h^{r j} R_{i j}-k^{i j} R_{i j}+\nabla^{i} \nabla^{j} k_{i j}-\nabla_{r} \nabla^{r} k_{s}^{s}\right. \\
& \quad-h^{i j}\left(\nabla_{r} \nabla_{i} h_{j}^{r}+\nabla_{i} \nabla_{r} h_{j}^{r}-\nabla_{r} \nabla^{r} h_{i j}-\nabla_{i} \nabla_{j} h_{s}^{s}\right) \\
& \quad-\nabla_{s} h_{r}^{s} \nabla_{a} h^{a r}+\nabla_{s} h^{s r} \nabla_{r} h_{a}^{a}-\frac{1}{4} \nabla_{r} h_{s}^{s} \nabla^{r} h_{a}^{a} \\
& \left.\quad+\frac{3}{4}\|\nabla h\|^{2}-\frac{1}{2} \nabla_{r} h_{s i} \nabla^{s} h^{r i}\right]+\left[t^{3}\right] .
\end{aligned}
$$

Let $(M, \eta, g)$ be a compact contact Riemannian manifold and let $\{g(t)\}$ be a curve in $\mathscr{M}(\eta)$ such that $g(0)=g$. We put $g(t)$ as $(13.1)$ and $\phi(t)$ as

$$
\phi_{j}^{i}(t)=\phi_{j}^{i}+t \varphi_{j}^{i}+t^{2} \Phi_{j}^{i}+\left[t^{3}\right] \text {. }
$$

Since the structure tensors $\{\phi(t), \eta, \xi, g(t)\}$ satisfy $(4.1) \sim(4.3)$, we obtain (4.6)-(4.8) and

$$
\begin{gathered}
k_{i r} \xi^{r}=\Phi_{r}^{i} \xi^{r}=\eta_{r} \Phi_{j}^{r}=0, \\
\Phi_{i j}+h_{i r} \varphi_{j}^{r}+k_{i r} \phi_{j}^{r}=0, \\
\phi_{r}^{i} \Phi_{j}^{r}+\varphi_{r}^{i} \varphi_{j}^{r}+\Phi_{r}^{i} \phi_{j}^{r}=0 .
\end{gathered}
$$

In $\S 4$, we verified $h_{i j}+h_{r s} \phi_{i}^{r} \phi_{j}^{s}=0$ and $h_{r}^{i} \phi_{j}^{r}=-\phi_{r}^{i} h_{j}^{r}$. Furthermore we get $\varphi_{i j}=\varphi_{j i}$ and

$$
\varphi_{i r} \varphi_{j}^{r}=h_{i r} h_{j}^{r}
$$

Operating $\phi_{k}^{j}$ to (13.7), we obtain $k_{i j}=\Phi_{i r} \phi_{j}^{r}+h_{i r} h_{j}^{r}$. Applying (13.8) and (13.9) to the last equality, we get $k_{i j}=-\phi_{i r} \Phi_{j}^{r}$, and hence $\Phi_{i j}=-\phi_{i}^{r} k_{r j}$. Therefore we get $k_{i j}+k_{r s} \phi_{i}^{r} \phi_{j}^{s}=h_{i r} h_{j}^{r}$. Summarizing the above we obtain the following.

Proposition 13.1. Let $(M, \eta, g)$ be a compact contact Riemannian manifold and let $\{g(t)\}$ be a curve in $\mathscr{M}(\eta)$ such that $g(0)=g$. If we put $g(t)$ as (13.1), then we get

$$
\phi_{j}^{i}(t)=\phi_{j}^{i}-t\left(h_{r}^{i} \phi_{j}^{r}\right)+t^{2}\left(\phi_{r}^{i} k_{j}^{r}\right)+\left[t^{3}\right]
$$

Furthermore $k_{i j}$ satisfies

$$
2 k_{i j}=k_{i j}-k_{r s} \phi_{i}^{r} \phi_{j}^{s}+h_{i r} h_{j}^{r}
$$

and hence $2 k_{r}^{r}=h^{r s} h_{r s}$.

Now we calculate the second derivative of $F(t)=F_{(\eta)}(g(t), f(t))$ at $t=0$ assuming that $F_{(\eta)}$ is critical at the pair $(g, f)$. 
Proposition 13.2. If $F_{(\eta)}$ is critical at $(g, f)$, then

$$
\begin{aligned}
\frac{d^{2} F}{d t^{2}}(0)= & 2 \int_{M}\left(-\mu \Delta_{P} \psi+{ }^{*} S \psi\right) \psi d M-2 c(p-1) \int_{M} f^{p-2} \psi^{2} d M \\
& -2 \int_{M}\left[2 \mu(h ; d f \otimes d \psi)-2\left(\nabla^{i} \nabla^{j} h_{i j}-h^{i j} R_{i j}\right) f \psi\right. \\
& \left.+\left(2 \nabla_{r} \nabla_{i} h_{j}^{r}-\nabla_{r} \nabla^{r} h_{i j}\right) \xi^{i} \xi^{j} f \psi\right] d M \\
+ & \int_{M}\left[2\|h\|^{2} f^{2}+h_{r}^{i} h^{r j} R_{i j} f^{2}+\mu h_{r}^{i} h^{r j} \nabla_{i} f \nabla_{j} f\right. \\
& -\left\{h^{i j} \nabla_{r} \nabla_{i} h_{j}^{r}+h^{i j} \nabla_{i} \nabla_{r} h_{j}^{r}+\nabla_{s} h_{r}^{s} \nabla_{i} h^{i r}+\frac{1}{2}\|\nabla h\|^{2}\right\} f^{2} \\
& +\left\{h^{r s}\left(2 \nabla_{r} \nabla_{i} h_{s j}-\nabla_{r} \nabla_{s} h_{i j}\right)+\frac{1}{2} \nabla_{i} h^{r s} \nabla_{j} h_{r s}\right. \\
- & \left.\left.-\nabla_{s} h_{i r} \nabla^{s} h_{j}^{r}+\nabla_{r} h_{s i} \nabla^{s} h_{j}^{r}\right\} \xi^{i} \xi^{j} f^{2}\right] d M .
\end{aligned}
$$

Proof. We put

$$
f(t)=f+t \psi+t^{2} \sigma+\left[t^{3}\right]
$$

Then differentiating (12.1) twice and using $h_{r}^{r}=0$ and $2 k_{r}^{r}=h^{r s} h_{r s}$, we obtain

$$
\begin{aligned}
& \frac{d^{2} F}{d t^{2}}(0)=\int_{M}\left[2 \mu\left(h_{r}^{i} h^{r j}-k^{i j}\right) \nabla_{i} f \nabla_{j} f-4 \mu(h ; d f \otimes d \psi)\right. \\
& +2 \mu\left(\|d \psi\|_{P}^{2}+2(d f ; d \sigma)_{P}\right) \\
& +2\left\{h_{r}^{i} h^{r j} R_{i j}-k^{i j} R_{i j}+\nabla^{i} \nabla^{j} k_{i j}-h^{i j} \nabla_{r} \nabla_{i} h_{j}^{r}-h^{i j} \nabla_{i} \nabla_{r} h_{j}^{r}\right. \\
& \left.-\nabla_{s} h_{r}^{s} \nabla_{a} h^{a r}-\frac{1}{4}\|\nabla h\|^{2}-\frac{1}{2} \nabla_{r} h_{s i} \nabla^{s} h^{r i}\right\} f^{2} \\
& -\frac{1}{2}\left\{4 \nabla_{r} \nabla_{i} k_{j}^{r}-2 \nabla_{r} \nabla^{r} k_{i j}-2 h^{r s}\left(2 \nabla_{r} \nabla_{i} h_{j s}-\nabla_{r} \nabla_{s} h_{i j}\right)\right. \\
& \left.-\nabla_{i} h^{r s} \nabla_{j} h_{r s}+2 \nabla_{s} h_{i r} \nabla^{s} h_{j}^{r}-2 \nabla_{r} h_{s i} \nabla^{s} h_{j}^{r}\right\} \xi^{i} \xi^{j} f^{2} \\
& +4\left(\nabla^{i} \nabla^{j} h_{i j}-h^{i j} R_{i j}\right) f \psi-2\left(2 \nabla_{r} \nabla_{i} h_{j}^{r}-\nabla_{r} \nabla^{r} h_{i j}\right) \xi^{i} \xi^{j} f \psi \\
& \left.+2^{*} S\left(\psi^{2}+2 f \sigma\right)\right] d M \text {, }
\end{aligned}
$$

where we have used $\xi^{i} \xi^{j} \nabla_{i} h_{r j}=-\xi^{i} \nabla_{i} \xi^{j} h_{r j}=0$, etc. Since

First we simplify terms in (13.12) which do not contain $\left(h_{i j}\right)$ nor $\left(k_{i j}\right)$.

$$
\frac{d^{2}}{d t^{2}}\left(\int_{M} f(t)^{p} d M\right)(0)=\int_{M}\left[p(p-1) f^{p-2} \psi^{2}+2 p f^{p-1} \sigma\right] d M=0
$$


we obtain

$$
\begin{aligned}
2 \int_{M} & {\left[\mu\left(\|d \psi\|_{P}^{2}+2(d f ; d \sigma)_{P}\right)+{ }^{*} S\left(\psi^{2}+2 f \sigma\right)\right] d M } \\
= & 2 \int_{M}\left[\left(-\mu \Delta_{P} \psi+{ }^{*} S \psi\right) \psi+2\left(-\mu \Delta_{P} f+{ }^{*} S f\right) \sigma\right] d M \\
& =2 \int_{M}\left[\left(-\mu \Delta_{P} \psi+{ }^{*} S \psi\right) \psi+2 c f^{p-1} \sigma\right] d M \\
& =2 \int_{M}\left[\left(-\mu \Delta_{P} \psi+{ }^{*} S \psi\right) \psi-c(p-1) f^{p-2} \psi^{2}\right] d M
\end{aligned}
$$

As for terms in (13.14) which contain $\left(k_{i j}\right)$, by the way analogous to (12.5) we obtain

$$
\begin{aligned}
-\int_{M} & {\left[2 \mu k^{i j} \nabla_{i} f \nabla_{j} f+2\left(k^{i j} R_{i j}-\nabla^{i} \nabla^{j} k_{i j}\right) f^{2}\right.} \\
& \left.+\left(2 \nabla_{r} \nabla_{i} k_{j}^{r}-\nabla_{r} \nabla^{r} k_{i j}\right) \xi^{i} \xi^{j} f^{2}\right] d M \\
= & \int_{M} 2 k^{i j} N_{i j} d M=\int_{M}\left(k^{i j}-k^{r s} \phi_{r}^{i} \phi_{s}^{j}+h_{r}^{i} h^{r j}\right) N_{i j}^{\prime} d M \\
= & \int_{M} 2 k^{i j} H_{i j} d M+\int_{M} h_{r}^{i} h^{r j} N_{i j}^{\prime} d M=\int_{M} h_{r}^{i} h^{r j} N_{i j} d M \\
= & \int_{M} h_{r}^{i} h^{r j}\left(2 f \nabla_{i} \nabla_{j} f+(2-\mu) \nabla_{i} f \nabla_{j} f-f^{2} R_{i j}+2 f^{2} g_{i j}\right) d M
\end{aligned}
$$

because $h_{r}^{i} h^{r j}$ satisfies $2 h_{r}^{i} h^{r j}=h_{r}^{a} h^{r b}\left(\delta_{a}^{i} \delta_{b}^{j}+\phi_{a}^{i} \phi_{b}^{j}\right)$ and hence

$$
\begin{gathered}
h_{r}^{i} h^{r j} \xi^{r} \nabla_{r}\left(f^{2} L_{\xi} g_{i j}\right)=0, \\
h_{r}^{i} h^{r j}\left(2 f^{2} \nabla_{a} \eta_{i} \phi_{j}^{a}\right)=h_{r}^{i} h^{r j}\left(2 f^{2} g_{i j}\right) .
\end{gathered}
$$

Applying these to (13.14) and using

$$
\begin{aligned}
2 \int_{M} & h_{r}^{i} h^{r j}\left(f \nabla_{i} \nabla_{j} f+\nabla_{i} f \nabla_{j} f\right) d M \\
& =\int_{M} h_{r}^{i} h^{r j} \nabla_{i} \nabla_{j} f^{2} d M \\
& =\int_{M}\left[\nabla_{j} \nabla_{i} h_{r}^{i} h^{r j}+\nabla_{i} h_{r}^{i} \nabla_{j} h^{r j}+\nabla_{j} h_{r}^{i} \nabla_{i} h^{r j}+h_{r}^{i} \nabla_{j} \nabla_{i} h^{r j}\right] f^{2} d M,
\end{aligned}
$$

we obtain the expression (13.11). Q.E.D.

Corollary 13.3. Suppose that ${ }^{*} S$ of a compact contact Riemannian manifold $(M, \eta, g)$ is constant. If $F_{(\eta, g)}$ is critical at $f=(\operatorname{vol}(M, g))^{-1 / p}$, then

$$
\frac{d^{2} F}{d t^{2}}(0)=2 \int_{M}\left(-\mu \Delta_{P} \psi+(2-p)^{*} S \psi\right) \psi d M
$$

Proof. By (12.8) we get ${ }^{*} S=c f^{p-2}$. Putting $h=0$ in (13.11), we obtain (13.13). Q.E.D. 
Corollary 13.4. Suppose that ${ }^{*} S$ of a compact contact Riemannian manifold $(M, \eta, g)$ is constant and that $\xi$ is a Killing vector field. If $F_{(\eta)}$ is critical at $(g, f)$, with $f=(\operatorname{vol}(M, g))^{-1 / p}$, then

$$
\begin{aligned}
\frac{d^{2} F}{d t^{2}}(0)= & 2 \int_{M}\left(-\mu \Delta_{P} \psi+(2-p)^{*} S \psi\right) \psi d M+ \\
+ & 4 f \int_{M}(h ; \nabla \nabla \psi) d M \\
& f^{2} \int_{M}\left[2\|h\|^{2}+h^{i j} h^{r s} R_{i r j s}+\nabla_{s} h_{r}^{s} \nabla_{i} h^{i r}+2 \xi^{i} \nabla_{i} h_{r j} h^{r s} \phi_{s}^{j}\right. \\
& \left.+\frac{1}{2}\left\|\nabla_{\xi} h\right\|^{2}-\frac{1}{2}\|\nabla h\|^{2}\right] d M .
\end{aligned}
$$

Proof. Since $\xi$ is a Killing vector field, operating $h^{i j}$ to (12.10), we obtain $h^{i j} R_{i j}=0$. By the Ricci identity we get

$$
\nabla_{r} \nabla_{i} h_{j}^{r}=\nabla_{i} \nabla_{r} h_{j}^{r}+R_{s i} h_{j}^{s}-R_{j r i}^{s} h_{s}^{r},
$$

and hence

$$
\begin{aligned}
-\int_{M} & {\left[h^{i j} \nabla_{r} \nabla_{i} h_{j}^{r}+h^{i j} \nabla_{i} \nabla_{r} h_{j}^{r}+\nabla_{s} h_{r}^{s} \nabla_{i} h^{i r}\right] d M } \\
& =-\int_{M}\left[2 h^{i j} \nabla_{i} \nabla_{r} h_{j}^{r}+h_{r}^{i} h^{r j} R_{i j}-h^{i j} h^{r s} R_{i r j s}+\nabla_{s} h_{r}^{s} \nabla_{i} h^{i r}\right] d M \\
& =\int_{M}\left[-h_{r}^{i} h^{r j} R_{i j}+h^{i j} h^{r s} R_{i r j s}+\nabla_{s} h_{r}^{s} \nabla_{i} h^{i r}\right] d M .
\end{aligned}
$$

Next, by $\nabla_{j} \xi^{i}=-\phi_{j}^{i}$, we obtain

$$
\begin{aligned}
& h^{r s}\left(2 \nabla_{r} \nabla_{i} h_{s j}-\nabla_{r} \nabla_{s} h_{i j}\right) \xi^{i} \xi^{j} \\
&= 2 h^{r s} \nabla_{i} h_{s j} \phi_{r}^{i} \xi^{j}+2 h^{r s} \nabla_{i} h_{s j} \xi^{i} \phi_{r}^{j}-2 h^{r s} h_{i j} \phi_{r}^{i} \phi_{s}^{j} \\
&= 2 h^{r s} h_{s j} \phi_{r}^{i} \phi_{i}^{j}+2 \xi^{i} \nabla_{i} h_{s j} h^{r s} \phi_{r}^{j}+2 h^{i j} h_{i j} \\
&= 2 \xi^{i} \nabla_{i} h_{s j} h^{r s} \phi_{r}^{j}, \\
&\left(2 \nabla_{r} \nabla_{i} h_{j}^{r}-\nabla_{r} \nabla^{r} h_{i j}\right) \xi^{i} \xi^{j}=0, \\
& \nabla_{s} h_{i r} \nabla^{s} h_{j}^{r} \xi^{i} \xi^{j}=h_{i r} h_{j}^{r} \phi_{s}^{i} \phi^{j s}=\|h\|^{2}, \\
& \nabla_{r} h_{s i} \nabla^{s} h_{j}^{r} \xi^{i} \xi^{j}=-h^{i s} h_{r j} \phi_{i}^{r} \phi_{s}^{j}=\|h\|^{2} .
\end{aligned}
$$

Applying these to (13.11), we obtain (13.14).

\section{UNIT SPHERES AS EXAMPLES}

Let $\left(S^{m}, g_{0}\right)$ be the unit sphere. By $\Delta$ we denote the Laplacian with respect to $g_{0}$. The $k$ th eigenvalue $\lambda_{k}$ of the Laplacian $\Delta$ acting on functions on the unit sphere $\left(S^{m}, g_{0}\right)$ is

$$
\lambda_{k}=k(m+k-1), \quad k=0,1,2, \ldots,
$$


with multiplicity $\theta\left(\lambda_{k}\right) ; \theta\left(\lambda_{0}\right)=1, \theta\left(\lambda_{1}\right)=m+1$ and

$$
\theta\left(\lambda_{k}\right)={ }_{m+k} C_{k}-{ }_{m+k-2} C_{k-2}, \quad k=2,3, \ldots
$$

Let $V_{k}$ denote the eigenspace corresponding to $\lambda_{k}$. If $m=2 n+1$, then $V_{k}$ has the following orthogonal decomposition:

$$
V_{k}=V_{k, k}+V_{k, k-2}+\cdots+V_{k, k-2[k / 2]},
$$

where $[k / 2]$ denotes the integral part of $k / 2$, and for $\psi \in V_{k, k-2 q}$,

$$
\xi \xi \psi+(k-2 q)^{2} \psi=0
$$

holds, where $\xi$ denotes the Killing vector field associated to a standard contact structure $\eta$ on $S^{m}$ (Tanno [14]).

As mentioned in Remark in $\S 12$, for $m=2 n+1, F_{\left(\eta, g_{0}\right)}$ is critical at $f_{0}=$ $\left(\operatorname{vol}\left(S^{m}, g_{0}\right)\right)^{-1 / p} .(13.13)$ implies

$$
\frac{d^{2} F}{d t^{2}}(0)=\frac{8(n+1)}{n} \int_{S^{m}}\left(-\Delta_{P} \psi-2 n \psi\right) \psi d S^{m} .
$$

We may call $\Psi$, defined by

$$
\Psi=-\Delta_{P}-2 n \mathrm{Id}=-\Delta+L_{\xi} L_{\xi}-2 n \mathrm{Id},
$$

the Jacobi operator corresponding to $F_{\left(\eta, g_{0}\right)}$. By (12.4), $\Psi$ acts on the space of functions $\psi$ on $S^{m}$ satisfying $\int_{S^{m}} \psi d S^{m}=0$.

Theorem 14.1. Let $\left(S^{m}, \eta, g_{0}\right), m=2 n+1$, be the unit sphere with a standard contact structure $\eta$, and let $\Psi$ be the Jacobi operator at $f_{0}=\left(\operatorname{vol}\left(S^{m}, g_{0}\right)\right)^{-1 / p}$ corresponding to $F_{\left(\eta, g_{0}\right)}$. Then, the stability of $F_{\left(\eta, g_{0}\right)}$ at $f_{0}$ (i.e., $d^{2} F(0) / d t^{2} \geq$ 0 ) is supported by

(i) $\Psi=0$ for $V_{1}$,

(ii) $\Psi$ is positive for $V_{k}, k \geq 2$.

Proof. Let $\psi \in V_{1}$. Then $\lambda_{1}=m$ and (14.2) give

$$
\Psi \psi=m \psi-\psi-2 n \psi=0 \text {. }
$$

Next, let $\psi \in V_{k, k-2 q}, k \geq 2$. Then (14.2) implies

$$
\Psi \psi=k(m+k-1) \psi-(k-2 q)^{2} \psi-2 n \psi,
$$

and the eigenspace decomposition by $\Psi$ corresponds to (14.1) canonically. Since

$$
k(m+k-1)-(k-2 q)^{2}-2 n \geq 2 n(k-1),
$$

$\Psi$ is positive for $V_{k}$ with $k \geq 2$. Q.E.D.

Next we consider $\left(S^{m}, g_{0}\right)$ with $m=4 r+3$. This space admits three Killing vector fields $\left\{\xi_{(1)}, \xi_{(2)}, \xi_{(3)}\right\}$, which are orthonormal and satisfy the following 
(cf. Tanno $[15,16]$, etc.)

$$
\begin{gathered}
{\left[\xi_{(\alpha)}, \xi_{(\beta)}\right]=2 \xi_{(\gamma)},} \\
\phi_{(\alpha)} \xi_{(\beta)}=-\phi_{(\beta)} \xi_{(\alpha)}=\xi_{(\gamma)}, \\
\phi_{(\alpha)} \phi_{(\beta)}-\xi_{(\alpha)} \otimes \eta_{(\beta)}=-\phi_{(\beta)} \phi_{(\alpha)}+\xi_{(\beta)} \otimes \eta_{(\alpha)}=\phi_{(\gamma)},
\end{gathered}
$$

where $\{\alpha, \beta, \gamma\}$ is a cyclic permutation of $\{1,2,3\}$, and $\phi_{(\alpha)}=-\nabla \xi_{(\alpha)}$ and $g_{0}\left(\xi_{(\alpha)}, X\right)=\eta_{(\alpha)}(X)$. Each $\eta_{(\alpha)}$ is a standard contact structure on $\left(S^{m}, g_{0}\right)$. We denote $g=g_{0}$ and $\xi=\xi_{(1)}$. Now we define $h$ by

$$
\begin{aligned}
h= & v\left(\eta_{(2)} \otimes \eta_{(2)}-\eta_{(3)} \otimes \eta_{(3)}\right) \\
& +w\left(\eta_{(2)} \otimes \eta_{(3)}+\eta_{(3)} \otimes \eta_{(2)}\right)
\end{aligned}
$$

where $v$ and $w$ are functions on $S^{m}$. Then

$$
h_{i r} \xi^{r}=0, \quad h_{i j}+h_{r s} \phi_{i}^{r} \phi_{j}^{s}=0
$$

are verified. We notice here that any $h$ defined by using only $\eta_{(2)}$ and $\eta_{(3)}$, and satisfying (14.7) is of the form (14.6).

Let $\psi=0$ or $\psi \in V_{1}$. Notice that $\psi \in V_{1}$ implies $\nabla \nabla \psi=-\psi g$. We consider the following deformation:

$$
g(t)_{i j}=g_{i j}+t h_{i j}+\left[t^{2}\right], \quad f(t)=f_{0}+t \psi+\left[t^{2}\right] .
$$

Then the first two terms of the right-hand side of (13.14) vanish. So, we calculate quantities in the third term of (13.14). First we obtain

$$
\|h\|^{2}=2\left(v^{2}+w^{2}\right) \text {. }
$$

Since $g_{0}$ is of constant curvature 1 , we get $h^{i j} h^{r s} R_{i r j s}=-\|h\|^{2}$. Operating $\nabla$ to $h_{i j}$, we obtain

$$
\begin{aligned}
\nabla_{r} h_{i j}= & v\left(\phi_{(2) r i} \eta_{(2) j}+\eta_{(2) i} \phi_{(2) r j}-\phi_{(3) r i} \eta_{(3) j}-\eta_{(3) i} \phi_{(3) r j}\right) \\
& +w\left(\phi_{(2) r i} \eta_{(3) j}+\eta_{(2) i} \phi_{(3) r j}+\phi_{(3) r i} \eta_{(2) j}+\eta_{(3) i} \phi_{(2) r j}\right) \\
& +\nabla_{r} v\left(\eta_{(2) i} \eta_{(2) j}-\eta_{(3) i} \eta_{(3) j}\right)+\nabla_{r} w\left(\eta_{(2) i} \eta_{(3) j}+\eta_{(3) i} \eta_{(2) j}\right)
\end{aligned}
$$

Therefore we obtain

$$
\nabla_{r} h_{j}^{r}=\left(\xi_{(2)} v+\xi_{(3)} w\right) \eta_{(2) j}+\left(-\xi_{(3)} v+\xi_{(2)} w\right) \eta_{(3) j}
$$

$$
\begin{aligned}
\int_{S^{m}} \nabla_{s} h_{r}^{s} \nabla_{i} h^{i r} d M=- & \int_{S^{m}}\left[v\left(\xi_{(2)} \xi_{(2)} v+\xi_{(3)} \xi_{(3)} v\right)\right. \\
& \left.+w\left(\xi_{(2)} \xi_{(2)} w+\xi_{(3)} \xi_{(3)} w\right)-4 w \cdot \xi v\right] d S^{m}, \\
\xi^{r} \nabla_{r} h_{i j}= & (2 v+\xi w)\left(\eta_{(2) i} \eta_{(3) j}+\eta_{(3) i} \eta_{(2) j}\right) \\
& +(-2 w+\xi v)\left(\eta_{(2) i} \eta_{(2) j}-\eta_{(3) i} \eta_{(3) j}\right),
\end{aligned}
$$




$$
\int_{S^{m}}\|\nabla h\|^{2} d M=\int_{S^{m}}\left[4(2 n+1)\left(v^{2}+w^{2}\right)-16 w \cdot \xi v-2 v \Delta v-2 w \Delta w\right] d M .
$$

Substituting (14.8)-(14.13) into (13.14) we obtain

$$
\begin{aligned}
\frac{d^{2} F}{d t^{2}}(0)=f_{0}^{2} \int_{S^{m}} & {\left[4(3-n)\left(v^{2}+w^{2}\right)-4 w \cdot \xi v\right.} \\
& +v\left(\Delta v-\xi \xi v-\xi_{(2)} \xi_{(2)} v-\xi_{(3)} \xi_{(3)} v\right) \\
& \left.+w\left(\Delta w-\xi \xi w-\xi_{(2)} \xi_{(2)} w-\xi_{(3)} \xi_{(3)} w\right)\right] d S^{m}
\end{aligned}
$$

Theorem 14.2. Let $\left(S^{m}, \eta, g_{0}\right), m=4 r+3$, be the unit sphere with a standard contact structure $\eta$. Then the instability of $F_{(\eta)}$ at the critical pair $\left(g_{0}, f_{0}\right)$, $f_{0}=\left(\operatorname{vol}\left(S^{m}, g_{0}\right)\right)^{-1 / p}$, is verified, for example, by the variation vector $(h, \psi)$, where

(i) $\psi=0$ or $\psi \in V_{1}$;

(ii) $h$ is defined by (14.6) with $v \in V_{k, k-2 q}$ and $w=(1 /(k-2 q)) \xi v$ such that

(a) $k-2 q \geq 5$, if $m=3$,

(b) $k-2 q \geq 1$, if $m \geq 7$.

Proof. We can see that the third and fourth terms in the integral of (14.14) are nonpositive, and, if $m=3$, they vanish. Let $v \in V_{k, k-2 q}$. Then $\xi \xi v=$ $-(k-2 q)^{2} v$, and $w=(1 /(k-2 q)) \xi v$ satisfies

$$
\begin{aligned}
\int_{S^{m}} w^{2} d S^{m} & =(k-2 q)^{-2} \int_{S^{m}}(\xi v)^{2} d S^{m} \\
& =(k-q)^{-2} \int_{S^{m}}(-v \xi \xi v) d S^{m}=\int_{S^{m}} v^{2} d S^{m} .
\end{aligned}
$$

Therefore, (14.14) gives

$$
\frac{d^{2} F}{d t^{2}}(0) \leq 4 f_{0}^{2} \int_{S^{m}}[2(3-n)-(k-2 q)] v^{2} d S^{m}
$$

(14.15) shows that the situation for $n \geq 3$ is somewhat different from the situation for $n=1$. If $n=1$, one needs to choose $k$ so that $k-2 q \geq 5$ for the negative second derivative. Q.E.D.

\section{REFERENCES}

1. D. E. Blair, Contact manifolds in Riemannian geometry, Lecture Notes in Math., vol. 509, Springer, Berlin, 1976.

2. _ Critical associated metrics on contact manifolds, J. Austral. Math. Soc. 37 (1984), 82-88. 
3. S. S. Chern and R. S. Hamilton, On Riemannian metrics adapted to three-dimensional contact manifolds, Lecture Notes in Math., vol. 1111, Springer, Berlin, 1985, pp. 279-305.

4. D. Jerison and J. M. Lee, A subelliptic, nonlinear eigenvalue problem and scalar curvature on CR manifolds, Contemp. Math., vol. 27, Amer. Math. Soc., Providence, R.I., 1984, pp. 57-63.

5. __ The Yamabe problem on CR manifolds, J. Differential Geometry 25 (1987), 167-197.

6. __ Extremals for the Sobolev inequality on the Heisenberg group and the CR Yamabe problem, preprint.

7. S. Sasaki, On differentiable manifolds with certain structures which are closely related to almost contact structure. I, Tôhoku Math. J. 12 (1960), 459-476.

8. S. Sasaki and Y. Hatakeyama, On differentiable manifolds with certain structures which are closely related to almost contact structure. II, Tôhoku Math. J. 13 (1961), 281-294.

9. N. Tanaka, On the pseudo-conformal geometry of hypersurfaces of the space of $n$ complex variables, J. Math. Soc. Japan 14 (1962), 387-429.

10. __, A differential geometric study on strongly pseudoconvex manifolds, Lectures in Math., vol. 9, Kyoto Univ., 1975.

11. __ On non-degenerate real hypersurfaces, graded Lie algebras and Cartan connections, Japan. J. Math. 2 (1976), 131-190.

12. S. Tanno, Harmonic forms and Betti numbers of certain contact Riemannian manifolds, J. Math. Soc. Japan 19 (1967), 308-316.

13. __ The topology of contact Riemannian manifolds, Illinois J. Math. 12 (1968), 700-714.

14. _ The first eigenvalue of the Laplacian on spheres, Tôhoku Math. J. 31 (1979), 179-185.

15. __ Some metrics on a (4r+3)-sphere and spectra, Tsukuba J. Math. 4 (1980), 99-105.

16. __ Geometric expressions of eigen 1-forms of the Laplacian on spheres, Spectra of Riemannian Manifolds, Kaigai, Tokyo, 1983, pp. 115-128.

17. S. M. Webster, On the pseudo-conformal geometry of a Kähler manifold, Math. Z. 157 (1977), 265-270.

18. $\ldots$, Pseudo-Hermitian structure on a real hypersurface, J. Differential Geometry 13 (1978), 25-41.

Department of Mathematics, Tokyo Institute of Technology, Ohokayama, Meguro, TOKYO, 152 JAPAN 